\title{
BIOLOGICAL CRYSTALLOGRAPHY
}

Volume 71 (2015)

Supporting information for article:

Selecting soluble/foldable protein domains through single gene- or genomic ORF-filtering: crystal structure of the head domain of Burkholderia pseudomallei antigen BPSL2063

Louise J. Gourlay, Clelia Peano, Cecilia Deantonio, Lucia Perletti, Alessandro Pietrelli, Riccardo Villa, Elena Matterazzo, Patricia Lassaux, Claudio Santoro, Simone Puccio, Daniele Sblattero and Martino Bolognesi 
Table S1 Details for the cloning and production of BPSL1626, BPSL1801, BPSL2520 and BPSL2063.

Restriction sites are highlighted in bold; the additional 5' CACC sequence necessary for topoisomerase cloning is underlined. Protein residues corresponding to vector cloning regions and fusion tags are indicated in italics.

DNA source: Burkholderia pseudomallei strain K96243

BPSL1626 Forward primer: CACCCAGACCGCGACGACCGGC

BPSL1626 Reverse primer: CTACTTGTACGTCAGCGCGAATACCGC

BPSL1801 Forward primer: CACCGCCGGCACCGGCACC

BPSL1801 Reverse primer: TTACTGGTAGCGAACCGTGAAGTTCGC

BPSL2063 ${ }_{\mathrm{D} 1}$ Forward primer: $\underline{\text { CACCCAAGCAGGGCAGAACGTG }}$

BPSL2063D1 Reverse primer: CTGATTTCAGAGCACGACGCTGTTGT

BPSL2063 2 Forward primer: CACCGCCGTGACCGCGCCGTCGTA

BPSL2063 2 Reverse primer: GATCGTCAGTCCTTGTCGACCTC

Expression vector: pET151/D-TOPO (Invitrogen)

Expression host: BL21 (DE3) Star E. coli cells

Complete amino acid sequence of the construct produced:

BPSL1626:

HHHHHHGKPIPNPLLGLDSTENLYFQGIDPFTQTATTGTINFTGSITDVPCEIDTAATSSNVTMAK VFANDFSGVGSTTGTTAFKIVLKNCGASTSGATVRFMGTTDSANPAALQTTAGGAGGVALQL VDDTGTPISIGSSSKAYTIAEGDNTFNFAARYIATSATVTGGAANATAVFALTYK BPSL1801:

HHHHHHGKPIPNPLLGLDSTENLYFQGIDPFTAGTGTLNFTGEIVAGACGIDAGSVDQTVRLGF VPANTFKAAGDKSTPQNFDIKLVDCDTSVAKNAYFTFTGTSNATQPKLIATVGSATNVGIRLQ SASGEYLDNGAEQKGPVVLSNGTSVARFAAMYESTAASVTPGTADGVANFTVRYQ BPSL2063D1: 
MRGSHHHHHHGMASMTGGQQMGRDLYDDDDKDHPFTQAGQNVNAIDTVSIGKQATASANDA IAIGTNTKASGPADIYMGLNAGAGAGSTTSPDGTVTLGIRNMGLGESAGSYVTGQNNTGIGYQ SGMNVTGDQNVGLGQQAGQFVTGTGNSAMGHLAGSTVSGSYNAAFGEYAGTNTSGGANAA FGFYAGRYINGTNNTALGAYDLPVVNGTWYGSYVTGSNNLGAGHNSGAYVSGASNVGLGD GAGTFVTGSNNVAIGTAAGSGAYTSGPSGATLNAALVASNTVSIGTRATASQSDAIAIGKGAT ASGAQSISIGTGNVVSGKGSGAIGDPSTVSGAGSYSIGNNNTVANSNTFVLGNGVTTTQDNSV VL

BPSL2063 2 :

MRGSHHHHHHGMASMTGGQQMGRDLYDDDDKDHPFTIDPKTGAVTAPSYTVYNADGTTSNV GNVGAAIDAINSTGIKYFHANSTKPDSQALGADSVAIGPNAVANNAGDVALGSGAVTSQAGG TLSETINGVTYSFAGTTPIGTVSVGAPGVERTITNVAAGRIGQSSTDAINGSQLYGTNQSIEALT DKMNSLGNTVANTLGSGASYNPQTGAVNGPANSGGVVTPTVIQEAANKWVSANPSTYVAPV ATGTNGMAVGSGAVSTGQNSVALGTNASDGGRSNVVSVGAPGAERQVTNVAAGTQATDAV NLGQMNGALAQQTDSFNQRLGAVQQDVDNVARAAYGGIAAATALTMIPEVDKD

BPSL2520 Forward primer: CATATGGCGCAATCGCTGTCGAACCAGA

BPSL2520 Reverse primer: GGATCCTTACTTGCCCGGCTTGACCG

Expression vector: pET14-b (Novagen)

Expression host: C41 (DE3) pLysS E. coli cells

Complete amino acid sequence of the construct produced:

MGSSHHHHHHSSGLVPRGSHMAQSLSNQTSA PAAAAPIDAD KKAAIKDLLD AIDAPKLVSA I ANSAEMQSK QLVPAILSDA LSENKTLNDK QKQAAVPTLQ KNAVPKLVDG AGKVFGTQQF TNDAMQAQYD AYAKYYSTSE IKDLTTFYKS PTGRKFIQVQ DQVGRDVVNG LMQKYMPQA IKATRDQADKE VAAVKPGK

BPSL2063 XTAL Forward primer: CGGGATCCATGGCGCCGACGACGCCCAACG

BPSL2063xtAL Reverse primer: GCGAATTCCCGCCGTTCATCTGCCCGAGGTTC

Expression vector: pET21-b (Novagen)

Expression host: BL21 (DE3) Star E. coli cells

Complete amino acid sequence of the construct produced: 
MASMTGGQQMGRGSMAPTTPNGGKPSQDLTLVGAASGPVALHNVAPGTASTDAVNVGQLGA VTTGLGGGAAIDPKTGAVTAPSYTVYNADGTTSNVGNVGAAIDAINSTGIKYFHANSTKPDSQ ALGADSVAIGPNAVANNAGDVALGSGAVTSQAGGTLSETINGVTYSFAGTTPIGTVSVGAPGV ERTITNVAAGRIGQSSTDAINGSQLYGTNQSIEALTDKMNSLGNTVANTLGSGASYNPQTGAV NGPANSGGVVTPTVIQEAANKWVSANPSTYVAPVATGTNGMAVGSGAVSTGQNSVALGTNA SDGGRSNVVSVGAPGAERQVTNVAAGTQATDAVNLGQMNGGNSSSVDKLAAALEHHHHHH 
Table S2 Crystallization of BPSL2063 XTAL

Method: Sitting drop vapour diffusion

Plate type: CrystalQuick 96 Well Sitting Drop Plate (Greiner)

Temperature: $293 \mathrm{~K}$

Protein concentration: $3.5 \mathrm{mg} / \mathrm{ml}$

Buffer composition of protein solution:

10 mM Tris- $\mathrm{HCl} \mathrm{pH} 8.0$

Composition of reservoir solution: 0.1M Malic acid, MES and Tris- $\mathrm{HCl}$ (MMT) buffer $\mathrm{pH}$ 4.0, $25 \%$

PEG1500

Volume and ratio of drop: $400 \mathrm{nl}$ (70\% protein)

Volume of reservoir: $100 \mu \mathrm{l}$ 
Table S3 Data collection and processing from a single BPSL2063 xTAL crystal

Values for the outer shell are given in parentheses.

\begin{tabular}{|c|c|}
\hline Diffraction source: & Beamline ID23-2, ESRF (Grenoble, France) \\
\hline Wavelength $(\AA)$ & 0.87290 \\
\hline Temperature $(\mathrm{K})$ & 100 \\
\hline Detector & CCD (MarMosaic 225) \\
\hline Crystal-detector distance (mm) & 223.94 \\
\hline Rotation range per image $\left(^{\circ}\right)$ & 0.65 \\
\hline Total rotation range $\left(^{\circ}\right)$ & 227.5 \\
\hline Space group & $\mathrm{P} 2_{1}$ \\
\hline$a, b, c(\AA)$ & $63.0,58.7,74.4$ \\
\hline$\alpha, \beta, \gamma\left({ }^{\circ}\right)$ & $90.0,104.690 .0$ \\
\hline Mosaicity $\left(^{\circ}\right)$ & 0.17 \\
\hline Resolution range $(\AA)$ & $40-1.8(1.9-1.8)$ \\
\hline Total No. of reflections & $224927(23959)$ \\
\hline No. of unique reflections & $48598(6612)$ \\
\hline Completeness (\%) & $99.0(93.3)$ \\
\hline Redundancy & $4.6(3.6)$ \\
\hline$\langle I / \sigma(I)\rangle$ & $14.8(2.6)$ \\
\hline$R_{\text {r.i.m. }}$ & $0.085(0.489)$ \\
\hline Overall $B$ factor from Wilson plot $\left(\AA^{2}\right)$ & 13.7 \\
\hline
\end{tabular}


Table S4 Structure solution and refinement for BPSL2063 XTAL

Values for the outer shell are given in parentheses.

\begin{tabular}{|c|c|}
\hline Resolution range $(\AA)$ & $39.7-1.8(1.8347-1.8001)$ \\
\hline Completeness (\%) & $98.9(88)$ \\
\hline$\sigma$ cutoff & 2 \\
\hline $\begin{array}{l}\text { No. of reflections, working } \\
\text { set }\end{array}$ & 46120 \\
\hline No. of reflections, test set & 2462 \\
\hline Final $R_{\text {cryst }}$ & 16.8 \\
\hline Final $R_{\text {free }}$ & 20.4 \\
\hline Cruickshank DPI & 0.18 \\
\hline \multicolumn{2}{|l|}{ No. of non-H atoms } \\
\hline Protein & 4291 \\
\hline Magnesium Ion & 7 \\
\hline Water & 448 \\
\hline Total & 4746 \\
\hline \multicolumn{2}{|l|}{ R.m.s. deviations } \\
\hline Bonds $(\AA)$ & 0.007 \\
\hline Angles $\left(^{\circ}\right)$ & 1.022 \\
\hline \multicolumn{2}{|l|}{ Average $B$ factors $\left(\AA^{2}\right)$} \\
\hline Protein & 20.6 \\
\hline Magnesium ions & 24.4 \\
\hline
\end{tabular}


Water

Ramachandran plot

Most favoured (\%)

Allowed (\%)
24.5

98.6

100 
Table S5 List of genes belonging to chromosome I with a mean_depth > 1 


\begin{tabular}{|c|c|c|c|c|c|c|c|c|}
\hline chr & start & end & locus_tag & strand & gene_coverage & mean_depth & peak_start & $\overline{\text { peak_end }}$ \\
\hline gi_52208053_emb_BX571965 & 3758107 & 3759573 & gltD & - & 0.43588 & 726.706 & 3758934 & 3759573 \\
\hline gi_52208053_emb_BX571965 & 1883679 & 1884209 & BPSL1626 & + & 1 & 342.309 & 1883679 & 1884209 \\
\hline gi_52208053_emb_BX571965 & 2220192 & 2220986 & BPSL1867 & + & 0.656171 & 109.177 & 2220192 & 2220713 \\
\hline gi_52208053_emb_BX571965 & 504599 & 505570 & BPSL0467 & + & 0.403708 & 84.7245 & 504599 & 504991 \\
\hline gi_52208053_emb_BX571965 & 3986567 & 3989494 & gcvP & + & 0.22412 & 69.9268 & 3988838 & 3989494 \\
\hline gi_52208053_emb_BX571965 & 2641055 & 2642704 & BPSL2200 & - & 0.136446 & 66.9911 & 2641055 & 2641280 \\
\hline gi_52208053_emb_BX571965 & 3008637 & 3009125 & $\operatorname{lrp}$ & - & 0.704918 & 64.1221 & 3008781 & 3009125 \\
\hline gi_52208053_emb_BX571965 & 3087604 & 3088743 & BPSL2559 & + & 0.341528 & 63.9126 & 3087604 & 3088394 \\
\hline gi_52208053_emb_BX571965 & 1732557 & 1733057 & BPSL1494 & - & 0.378 & 60.4444 & 1732868 & 1733057 \\
\hline gi_52208053_emb_BX571965 & 503530 & 504348 & BPSL0466 & + & 1 & 54.3301 & 503530 & 504348 \\
\hline gi_52208053_emb_BX571965 & 2640218 & 2640919 & BPSL2199 & - & 0.850214 & 48.4648 & 2640323 & 2640919 \\
\hline gi_52208053_emb_BX571965 & 3086825 & 3087088 & BPSL2558 & + & 0.395437 & 44.6635 & 3086984 & 3087088 \\
\hline gi_52208053_emb_BX571965 & 223564 & 223818 & BPSL0214 & + & 0.555118 & 43.1844 & 223677 & 223818 \\
\hline gi_52208053_emb_BX571965 & 1724107 & 1724802 & BPSL1486 & - & 0.351079 & 43.0697 & 1724558 & 1724802 \\
\hline gi_52208053_emb_BX571965 & 3714325 & 3714939 & BPSL3109 & + & 0.285016 & 35.3771 & 3714325 & 3714500 \\
\hline gi_52208053_emb_BX571965 & 481272 & 482030 & BPSL0441 & - & 0.653034 & 33.7434 & 481294 & 481789 \\
\hline gi_52208053_emb_BX571965 & 2282088 & 2283647 & emrB & + & 0.118024 & 32.6576 & 2283345 & 2283529 \\
\hline gi_52208053_emb_BX571965 & 2219044 & 2220072 & pyrD & + & 0.213035 & 31.242 & 2219853 & 2220072 \\
\hline gi_52208053_emb_BX571965 & 2804238 & 2805227 & BPSL2322 & - & 0.651163 & 30.1134 & 2804345 & 2804989 \\
\hline gi_52208053_emb_BX571965 & 2143834 & 2144346 & BPSL1801 & - & 0.814453 & 29.7986 & 2143929 & 2144346 \\
\hline gi_52208053_emb_BX571965 & 2162248 & 2163822 & BPSL1815 & - & 0.495553 & 29.7359 & 2162543 & 2163323 \\
\hline gi_52208053_emb_BX571965 & 223950 & 224993 & BPSL0215 & + & 0.439118 & 29.3341 & 223950 & 224408 \\
\hline gi_52208053_emb_BX571965 & 3713279 & 3713950 & BPSL3108 & - & 0.704918 & 29.186 & 3713477 & 3713950 \\
\hline gi_52208053_emb_BX571965 & 1023539 & 1024744 & BPSL0882 & - & 0.248963 & 25.6933 & 1023539 & 1023839 \\
\hline gi_52208053_emb_BX571965 & 1299323 & 1301113 & BPSL1118 & + & 0.506145 & 24.1843 & 1299687 & 1300727 \\
\hline gi_52208053_emb_BX571965 & 1804461 & 1805555 & potF & + & 0.456124 & 23.7756 & 1804461 & 1804960 \\
\hline gi_52208053_emb_BX571965 & 3439438 & 3440016 & BPSL2874 & - & 0.99481 & 22.647 & 3439441 & 3440016 \\
\hline gi_52208053_emb_BX571965 & 1733194 & 1734456 & rho & - & 0.16878 & 21.2207 & 1733194 & 1733407 \\
\hline gi_52208053_emb_BX571965 & 794567 & 795328 & BPSL0697 & - & 0.609724 & 20.5453 & 794864 & 795328 \\
\hline gi_52208053_emb_BX571965 & 1961961 & 1963346 & BPSL1681 & + & 0.32491 & 18.7022 & 1962097 & 1962547 \\
\hline gi_52208053_emb_BX571965 & 3764662 & 3765375 & BPSL3160 & - & 0.535764 & 18.4869 & 3764993 & 3765375 \\
\hline gi_52208053_emb_BX571965 & 4051535 & 4052692 & BPSL3412 & - & 0.313742 & 18.2397 & 4052329 & 4052692 \\
\hline gi_52208053_emb_BX571965 & 3617871 & 3619031 & BPSL3036 & + & 0.357759 & 18.012 & 3617871 & 3618286 \\
\hline gi_52208053_emb_BX571965 & 1308976 & 1309356 & BPSL1125 & + & 0.0131579 & 18 & 1308976 & 1308981 \\
\hline gi_52208053_emb_BX571965 & 1581044 & 1581547 & BPSL1353 & + & 0.856859 & 17.9118 & 1581044 & 1581475 \\
\hline gi_52208053_emb_BX571965 & 3021173 & 3022075 & cysM & - & 0.568736 & 17.4951 & 3021562 & 3022075 \\
\hline gi_52208053_emb_BX571965 & 2247791 & 2249182 & BPSL1887 & - & 0.140187 & 16.4923 & 2247791 & 2247986 \\
\hline gi_52208053_emb_BX571965 & 3731572 & 3732354 & tatC & - & 0.703325 & 16.2491 & 3731603 & 3732153 \\
\hline gi_52208053_emb_BX571965 & 2843904 & 2846192 & BPSL2351 & + & 0.189685 & 16.2189 & 2843904 & 2844338 \\
\hline gi_52208053_emb_BX571965 & 4020565 & 4021704 & BPSL3388 & - & 0.102722 & 15.7094 & 4021587 & 4021704 \\
\hline gi_52208053_emb_BX571965 & 1725071 & 1725565 & BPSL1487 & + & 0.159919 & 15.6203 & 1725071 & 1725273 \\
\hline gi_52208053_emb_BX571965 & 1262824 & 1264374 & BPSL1093 & + & 0.143871 & 15.1345 & 1263554 & 1263777 \\
\hline gi_52208053_emb_BX571965 & 3879171 & 3879950 & BPSL3263 & - & 1 & 14.896 & 3879171 & 3879950 \\
\hline gi_52208053_emb_BX571965 & 4013381 & 4014175 & BPSL3383 & - & 0.0944584 & 14.6933 & 4014056 & 4014131 \\
\hline gi_52208053_emb_BX571965 & 3036533 & 3039133 & gyrA & - & 0.193846 & 14.6171 & 3036533 & 3037037 \\
\hline gi_52208053_emb_BX571965 & 2271756 & 2274620 & sucA & - & 0.257682 & 14.2561 & 2272662 & 2273400 \\
\hline gi_52208053_emb_BX571965 & 958008 & 958883 & BPSL0824 & - & 0.008 & 14 & 958876 & 958883 \\
\hline gi_52208053_emb_BX571965 & 2863293 & 2864489 & BPSL2368 & + & 0.359532 & 13.9442 & 2863293 & 2863723 \\
\hline gi_52208053_emb_BX571965 & 497056 & 497769 & BPSL0459 & + & 0.2777 & 13.702 & 497056 & 497254 \\
\hline gi_52208053_emb_BX571965 & 602532 & 606566 & BPSL0548 & + & 0.144769 & 13.4589 & 604251 & 606566 \\
\hline gi_52208053_emb_BX571965 & 359487 & 361604 & plcN2 & - & 0.348607 & 13.3252 & 360861 & 361599 \\
\hline gi_52208053_emb_BX571965 & 138897 & 139754 & BPSL0124 & + & 0.123687 & 13.1132 & 138897 & 139003 \\
\hline gi_52208053_emb_BX571965 & 4041903 & 4043054 & BPSL3404 & - & 0.338836 & 12.7974 & 4042163 & 4042553 \\
\hline gi_52208053_emb_BX571965 & 3696333 & 3696911 & BPSL3096 & + & 0.178201 & 12.699 & 3696333 & 3696436 \\
\hline gi_52208053_emb_BX571965 & 3022087 & 3022398 & BPSL2507A & - & 0.115756 & 12.4722 & 3022087 & 3022123 \\
\hline gi_52208053_emb_BX571965 & 2514194 & 2514919 & ompR & + & 0.481379 & 12.4097 & 2514194 & 2514543 \\
\hline
\end{tabular}


gi_52208053_emb_BX571965 gi_52208053_emb_BX571965 gi_52208053_emb_BX571965 gi_52208053_emb_BX571965 gi_52208053_emb_BX571965 gi_52208053_emb_BX571965 gi_52208053_emb_BX571965 gi_52208053_emb_BX571965 gi_52208053_emb_BX571965 gi_52208053_emb_BX571965 gi_52208053_emb_BX571965 gi_52208053_emb_BX571965 gi_52208053_emb_BX571965 gi_52208053_emb_BX571965 gi_52208053_emb_BX571965 gi_52208053_emb_BX571965 gi_52208053_emb_BX571965 gi_52208053_emb_BX571965 gi_52208053_emb_BX571965 gi_52208053_emb_BX571965 gi_52208053_emb_BX571965 gi_52208053_emb_BX571965 gi_52208053_emb_BX571965 gi_52208053_emb_BX571965 gi_52208053_emb_BX571965 gi_52208053_emb_BX571965 gi_52208053_emb_BX571965 gi_52208053_emb_BX571965 gi_52208053_emb_BX571965 gi_52208053_emb_BX571965 gi_52208053_emb_BX571965 gi_52208053_emb_BX571965 gi_52208053_emb_BX571965 gi_52208053_emb_BX571965 gi_52208053_emb_BX571965 gi_52208053_emb_BX571965 gi_52208053_emb_BX571965 gi_52208053_emb_BX571965 gi_52208053_emb_BX571965 gi_52208053_emb_BX571965 gi_52208053_emb_BX571965 gi_52208053_emb_BX571965 gi_52208053_emb_BX571965 gi_52208053_emb_BX571965 gi_52208053_emb_BX571965 gi_52208053_emb_BX571965 gi_52208053_emb_BX571965 gi_52208053_emb_BX571965 gi_52208053_emb_BX571965 gi_52208053_emb_BX571965 gi_52208053_emb_BX571965 gi_52208053_emb_BX571965 gi_52208053_emb_BX571965 gi_52208053_emb_BX571965 gi_52208053_emb_BX571965 gi_52208053_emb_BX571965 gi_52208053_emb_BX571965 gi_52208053_emb_BX571965
40530134054248 BPSL3413 14471361448863 BPSL1253 12215241221904 BPSL1053 25136342513972 BPSL2093 21043292114201 BPSL1778 17528381754178 hisS 959141959605 BPSL0825 $92536 \quad 93765$ intA

21566522157467 BPSL1809 19530751954175 BPSL1674 29610302962328 BPSL2455 11984371199537 BPSL1029 38614073862237 BPSL3249 32384013239633 BPSL2708 $107334 \quad 107894$ speG 19544661954945 BPSL1675 20990612104307 BPSL1777 22604932260663 BPSL1899 14166511417913 BPSL1233 124499124783 BPSL0112 607232607906 BPSL0550 25667682569167 ppsA 32617863262568 BPSL2726 25967112598786 ligA 21747842176310 BPSL1826 40112014011632 BPSL3379 12666561267873 BPSL1096 $36484163649153 \mathrm{dsbC}$ 28420002843166 BPSL2350 16759321676660 BPSL1440 690580693099 BPSL0611 17542311754860 BPSL1515 606593607069 BPSL0549A 19580401959680 BPSL1678 124780125262 BPSL0113 8408585188 dnaN

38801263882111 BPSL3264 442663443874 BPSL0408 15805681581044 greA 11647231165262 BPSL1001 407612409198 BPSL0376 $21523092152980 \mathrm{amrR}$ 21717062172752 BPSL1824 36347483636046 paaA 33975393399035 tldD $327333 \quad 330545$ silA 25825952583986 BPSL2152 22469872247718 BPSL1886 $324453 \quad 325754$ BPSL0307 25916712594247 BPSL2161 648080649468 BPSL0584 496759496968 BPSL0458a 38601373861387 BPSL3248 35884183589029 coaE 166687167049 BPSL0155 18843081885078 BPSL1627 $\begin{array}{lll}1767550 & 1769874 \text { tex }\end{array}$ 34654633467028 purH

.

\begin{tabular}{|c|c|c|c|}
\hline 0.0890688 & 12.0364 & 4053013 & 4053123 \\
\hline 0.0127389 & 12 & 1447930 & 1447952 \\
\hline 0.315789 & 11.8833 & 1221784 & 1221904 \\
\hline 0.363905 & 11.7154 & 2513849 & 2513972 \\
\hline 0.0409238 & 11.5594 & 2105943 & 2112636 \\
\hline 0.0820896 & 11.3091 & 1754068 & 1754178 \\
\hline 0.575431 & 10.5993 & 959141 & 959408 \\
\hline 0.389748 & 10.5658 & 93127 & 93606 \\
\hline 0.47362 & 10.3264 & 2157081 & 2157467 \\
\hline 0.337273 & 10.0135 & 1953804 & 1954175 \\
\hline 0.224961 & 9.76027 & 2961866 & 2962158 \\
\hline 0.108182 & 9.31933 & 1199418 & 1199537 \\
\hline 0.428916 & 9.2809 & 3861407 & 3861763 \\
\hline 0.452922 & 9.25269 & 3238401 & 3238959 \\
\hline 0.742857 & 9.04327 & 107478 & 107894 \\
\hline 0.334029 & 8.75 & 1954466 & 1954626 \\
\hline 0.00686237 & 8.72222 & 2101271 & 2101307 \\
\hline 1 & 8.47647 & 2260493 & 2260663 \\
\hline 0.310618 & 8.2398 & 1416651 & 1417043 \\
\hline 0.302817 & 8 & 124697 & 124783 \\
\hline 0.0296736 & 8 & 607232 & 607252 \\
\hline 0.142559 & 7.98538 & 2568825 & 2569167 \\
\hline 0.450128 & 7.8267 & 3262216 & 3262568 \\
\hline 0.269398 & 7.78891 & 2598167 & 2598726 \\
\hline 0.250983 & 7.6658 & 2175342 & 2175725 \\
\hline 0.62877 & 7.66421 & 4011361 & 4011632 \\
\hline 0.193098 & 7.53617 & 1266885 & 1267120 \\
\hline 0.333786 & 7.51626 & 3648907 & 3649153 \\
\hline 0.0746141 & 7.48276 & 2843079 & 2843166 \\
\hline 0.0769231 & 7.48214 & 1675932 & 1675988 \\
\hline 0.169115 & 7.29577 & 691681 & 692107 \\
\hline 0.489666 & 7.25 & 1754231 & 1754539 \\
\hline 1 & 7.22899 & 606593 & 607069 \\
\hline 0.2 & 7.05488 & 1959164 & 1959492 \\
\hline 1 & 7.0332 & 124780 & 125262 \\
\hline 0.517679 & 6.94046 & 84377 & 84948 \\
\hline 0.0277078 & 6.92727 & 3880126 & 3880181 \\
\hline 0.223782 & 6.87823 & 443603 & 443874 \\
\hline 0.201681 & 6.86458 & 1580948 & 1581044 \\
\hline 0.131725 & 6.77465 & 1164821 & 1164892 \\
\hline 0.113493 & 6.76667 & 409018 & 409198 \\
\hline 0.308495 & 6.76329 & 2152309 & 2152516 \\
\hline 0.564054 & 6.74407 & 2171943 & 2172533 \\
\hline 0.410632 & 6.64916 & 3635393 & 3635926 \\
\hline 0.316176 & 6.62791 & 3398504 & 3399035 \\
\hline 0.130448 & 6.58711 & 327333 & 330545 \\
\hline 0.179008 & 6.51807 & 2582595 & 2582844 \\
\hline 0.556772 & 6.37838 & 2247311 & 2247718 \\
\hline 0.269024 & 6.37429 & 324700 & 325050 \\
\hline 0.173525 & 6.3736 & 2593032 & 2593479 \\
\hline 0.154899 & 6.37209 & 649253 & 649468 \\
\hline 0.794258 & 6.36747 & 496802 & 496968 \\
\hline 0.2576 & 6.35404 & 3861065 & 3861387 \\
\hline 0.235679 & 6.30556 & 3588418 & 3588562 \\
\hline 0.779006 & 6.2234 & 166767 & 167049 \\
\hline 0.790909 & 6.15435 & 1884308 & 1885078 \\
\hline 0.26506 & 6.14935 & 1767672 & 1768310 \\
\hline 0.208946 & 6.01223 & 3465705 & 3466032 \\
\hline
\end{tabular}


gi_52208053_emb_BX571965 gi_52208053_emb_BX571965 gi_52208053_emb_BX571965 gi_52208053_emb_BX571965 gi_52208053_emb_BX571965 gi_52208053_emb_BX571965 gi_52208053_emb_BX571965 gi_52208053_emb_BX571965 gi_52208053_emb_BX571965 gi_52208053_emb_BX571965 gi_52208053_emb_BX571965 gi_52208053_emb_BX571965 gi_52208053_emb_BX571965 gi_52208053_emb_BX571965 gi_52208053_emb_BX571965 gi_52208053_emb_BX571965 gi_52208053_emb_BX571965 gi_52208053_emb_BX571965 gi_52208053_emb_BX571965 gi_52208053_emb_BX571965 gi_52208053_emb_BX571965 gi_52208053_emb_BX571965 gi_52208053_emb_BX571965 gi_52208053_emb_BX571965 gi_52208053_emb_BX571965 gi_52208053_emb_BX571965 gi_52208053_emb_BX571965 gi_52208053_emb_BX571965 gi_52208053_emb_BX571965 gi_52208053_emb_BX571965 gi_52208053_emb_BX571965 gi_52208053_emb_BX571965 gi_52208053_emb_BX571965 gi_52208053_emb_BX571965 gi_52208053_emb_BX571965 gi_52208053_emb_BX571965 gi_52208053_emb_BX571965 gi_52208053_emb_BX571965 gi_52208053_emb_BX571965 gi_52208053_emb_BX571965 gi_52208053_emb_BX571965 gi_52208053_emb_BX571965 gi_52208053_emb_BX571965 gi_52208053_emb_BX571965 gi_52208053_emb_BX571965 gi_52208053_emb_BX571965 gi_52208053_emb_BX571965 gi_52208053_emb_BX571965 gi_52208053_emb_BX571965 gi_52208053_emb_BX571965 gi_52208053_emb_BX571965 gi_52208053_emb_BX571965 gi_52208053_emb_BX571965 gi_52208053_emb_BX571965 gi_52208053_emb_BX571965 gi_52208053_emb_BX571965 gi_52208053_emb_BX571965 gi_52208053_emb_BX571965
27640772765156 BPSL2297 $757548 \quad 759647$ glyS

11532731157391 BPSL0995 37081833710009 BPSL3103 39744013974607 BPSL3351 138152138787 BPSL0123 14160761416558 BPSL1231 39482633949327 BPSL3329 24596722460508 BPSL2055 676682679045 BPSL0602 928931930127 purK

12427751243737 BPSL1074 556694558334 BPSL0507 40022184003627 BPSL3373 25426802543693 BPSL2116 $31351513135831 \mathrm{pgl}$ 34967633498214 purB 491323492900 BPSL0453 22688142270244 odhL 24435352446870 BPSL2046 35835873584882 BPSL3007 37655683766299 BPSL3161 581696582589 BPSL0529 16179231618318 BPSL1390 38703993870716 BPSL3255 25070622507385 BPSL2087 25802212582530 BPSL2151 40647694065299 BPSL3422 12220771222616 eco 40566564057852 BPSL3416 $585023 \quad 586531 \mathrm{rpoN}$ 38586083860011 BPSL3247 453402455528 BPSL0419 10397481041862 fusA 35874853588414 gspO 938273939691 BPSL0808 14102001411672 nuoN 22883732289848 nusA 13207251322113 BPSL1136 40439674044761 BPSL3406 40117324012373 BPSL3380 23220432322948 BPSL1950 19178341918952 BPSL1655 649491649814 BPSL0585 $788147 \quad 789820$ BPSL0692 325765327336 BPSL0308

$8395 \quad 10668$ gspD 482135483709 BPSL0442 297527298126 BPSL0284 335475336470 BPSL0314 951272952696 BPSL0818 34601033460711 BPSL2891 40546584055635 BPSL3414 26163422617412 alr 20891482090209 hoxN 25770912577879 lpxA 13967911397150 nuoA 37262513727009 petC
0.134384

0.011434

0.0531812

0.117744

0.898058

0.92126

0.338174

0.379699

0.598086

0.186627

0.496656

0.75052

0.432317

0.300923

0.452122

0.2

0.0303239

0.436271

0.16993

0.22099

0.400772

0.573187

0.567749

0.36962

0.55836

0.544892

0.389779

0.0792453

0.44898

0.048495

0.234085

0.645046

0.0719661

0.228477

0.345533

0.284908

0.0774457

0.238644

0.0850144

0.115869

0.422777

0.358011

0.242397

0.74613

0.0723252

0.189052

0.161461

0.257942

0.717863

0.205025

0.143961

0.679276

0.132037

0.00186916

0.206409

0.28934

0.445682

0.166227
27650112765156

$757548 \quad 757572$

$\begin{array}{llr}5.94977 & 1157098 & 1157317\end{array}$

$\begin{array}{llll}5.94884 & 3708254 & 3708469\end{array}$

$\begin{array}{lll}5.82162 & 3974422 & 3974607\end{array}$

$\begin{array}{lll}5.8 & 138152 & 138787\end{array}$

$\begin{array}{llll}5.78528 & 1416395 & 1416558\end{array}$

$\begin{array}{llll}5.76485 & 3948556 & 3948960\end{array}$

$\begin{array}{lll}5.756 & 2459875 & 2460375\end{array}$

$\begin{array}{lll}5.67347 & 677749 & 678862\end{array}$

$\begin{array}{lll}5.67003 & 929168 & 929843\end{array}$

$\begin{array}{lll}5.63573 & 1242851 & 1243573\end{array}$

$\begin{array}{rrr}5.5275 & 557157 & 558022\end{array}$

$\begin{array}{llll}5.40566 & 4002474 & 4002898\end{array}$

$\begin{array}{lll}5.36026 & 2542885 & 2543343\end{array}$

$\begin{array}{llll}5.20588 & 3135304 & 3135440\end{array}$

$\begin{array}{lll}5.18182 & 3497572 & 3497616\end{array}$

$\begin{array}{lll}5.17587 & 491496 & 492900\end{array}$

$\begin{array}{lll}5.16461 & 2268814 & 2269057\end{array}$

$\begin{array}{lll}5.16147 & 2443721 & 2445684\end{array}$

$\begin{array}{llll}5.13487 & 3584331 & 3584850\end{array}$

$\begin{array}{llll}5.09547 & 3765568 & 3765987\end{array}$

$\begin{array}{lll}5.0572 & 581817 & 582324\end{array}$

$1618172 \quad 1618318$

$3870399 \quad 3870576$

$\begin{array}{llll}4.98864 & 2507153 & 2507329\end{array}$

$\begin{array}{llll}4.95667 & 2581630 & 2582530\end{array}$

$\begin{array}{llll}4.85714 & 4065048 \quad 4065090\end{array}$

$\begin{array}{llll}4.84711 & 1222077 & 1222319\end{array}$

$\begin{array}{llll}4.82759 & 4057159 & 4057217\end{array}$

$\begin{array}{lll}4.79037 & 585529 & 586345\end{array}$

$\begin{array}{lll}4.74033 & 3858608 \quad 3859996\end{array}$

$\begin{array}{lll}4.56209 & 453402 \quad 453555\end{array}$

$\begin{array}{lll}4.53002 & 1041307 & 1041790\end{array}$

$\begin{array}{llll}4.47664 & 3588093 & 3588414\end{array}$

$\begin{array}{lll}4.41584 & 938823 & 939621\end{array}$

$\begin{array}{llll}4.39474 & 1410532 & 1410646\end{array}$

$\begin{array}{lll}4.3608 & 2288373 & 2288725\end{array}$

$\begin{array}{llll}4.32203 & 1321632 & 1322113\end{array}$

$4.29348 \quad 4044508 \quad 4044600$

$\begin{array}{lll}4.28782 & 4011732 & 4012003\end{array}$

$\begin{array}{lll}4.25309 & 2322412 & 2322736\end{array}$

$\begin{array}{lll}4.24723 \quad 1917834 & 1918105\end{array}$

$\begin{array}{lll}4.24066 & 649491 \quad 649732\end{array}$

$\begin{array}{lll}4.19835 & 788147 & 788268\end{array}$

$\begin{array}{lll}4.14478 & 327039 & 327336\end{array}$

$\begin{array}{lrr}4.11989 & 8395 & 8762\end{array}$

$\begin{array}{lll}4.0936 & 482402 \quad 483709\end{array}$

$\begin{array}{lll}4.07674 & 297641 \quad 298084\end{array}$

$335867 \quad 336071$

$951611 \quad 951816$

$3460103 \quad 3460516$

$4055506 \quad 4055635$

$2617410 \quad 2617412$

$2089749 \quad 2089968$

$\begin{array}{ll}2577166 & 2577394\end{array}$

$\begin{array}{ll}1396791 & 1396951\end{array}$

$3726251 \quad 3726377$ 
gi_52208053_emb_BX571965 gi_52208053_emb_BX571965 gi_52208053_emb_BX571965 gi_52208053_emb_BX571965 gi_52208053_emb_BX571965 gi_52208053_emb_BX571965 gi_52208053_emb_BX571965 gi_52208053_emb_BX571965 gi_52208053_emb_BX571965 gi_52208053_emb_BX571965 gi_52208053_emb_BX571965 gi_52208053_emb_BX571965 gi_52208053_emb_BX571965 gi_52208053_emb_BX571965 gi_52208053_emb_BX571965 gi_52208053_emb_BX571965 gi_52208053_emb_BX571965 gi_52208053_emb_BX571965 gi_52208053_emb_BX571965 gi_52208053_emb_BX571965 gi_52208053_emb_BX571965 gi_52208053_emb_BX571965 gi_52208053_emb_BX571965 gi_52208053_emb_BX571965 gi_52208053_emb_BX571965 gi_52208053_emb_BX571965 gi_52208053_emb_BX571965 gi_52208053_emb_BX571965 gi_52208053_emb_BX571965 gi_52208053_emb_BX571965 gi_52208053_emb_BX571965 gi 52208053 emb_BX571965 gi_52208053_emb_BX571965 gi_52208053_emb_BX571965 gi_52208053_emb_BX571965 gi_52208053_emb_BX571965 gi_52208053_emb_BX571965 gi_52208053_emb_BX571965 gi_52208053_emb_BX571965 gi_52208053_emb_BX571965 gi_52208053_emb_BX571965 gi_52208053_emb_BX571965 gi_52208053_emb_BX571965 gi_52208053_emb_BX571965 gi_52208053_emb_BX571965 gi_52208053_emb_BX571965 gi_52208053_emb_BX571965 gi_52208053_emb_BX571965 gi 52208053 emb_BX571965 gi_52208053_emb_BX571965 gi_52208053_emb_BX571965 gi_52208053_emb_BX571965 gi_52208053_emb_BX571965 gi_52208053_emb_BX571965 gi_52208053_emb_BX571965 gi_52208053_emb_BX571965 gi_52208053_emb_BX571965 gi_52208053_emb_BX571965
$37922843794167 \mathrm{dsbD}$

19162301917690 gabD

31780843178947 BPSL2654

38675163867743 BPSL3254A

18444971845597 BPSL1591

330542330883 BPSL0310

18027681804075 BPSL1554

409296409979 BPSL0377

36492463650427 BPSL3059

18124591813862 BPSL1562

$187471 \quad 189927 \mathrm{mrdA}$

22461862246836 BPSL1885A

39330373933741 aqpZ

23721022372983 BPSL1990

34112693412264 phnD

24679782471250 BPSL2063

751131752180 pdxA

349356350318 BPSL0327

$551745 \quad 553340$ cydA

39746913974993 BPSL3352

38517223853992 BPSL3242

10215021022770 BPSL0881

15693651571026 BPSL1345

40556324056516 BPSL3415

27829662784246 BPSL2307

33012563302281 tolA

376064377755 BPSL0353

38886773889477 BPSL3272

32353403236938 BPSL2706

302370303203 BPSL0290

11761471177136 BPSL1010

10638731065069 BPSL0911

14086851410175 nuoM

32376753238121 BPSL2707

36597043661371 BPSL3067

34223013423251 ubiA

$834328 \quad 835428$ BPSL0725

16407471642681 BPSL1410

838970840907 BPSL0729

29302862931314 BPSL2424

20779792079352 BPSL1763

30358383036434 BPSL2520

160948162120 BPSL0150

206377207180 BPSL0199

$654016 \quad 660111$ BPSL0590

14822631482442 BPSL1279

17193331719875 BPSL1482

20503802051318 BPSL1739

21612562162251 BPSL1814

26637182664812 BPSL2219

30068693007336 BPSL2494

34400833440643 BPSL2875

37716263772858 BPSL3167

$572627 \quad 573232$ pth

$21930332194016 \mathrm{sbp}$

23595842362451 valS

$186311 \quad 187459$ rodA

668159672238 BPSL0597
-

\begin{tabular}{|c|c|c|c|}
\hline 0.0902815 & 3.98824 & 3793900 & 3794070 \\
\hline 0.366438 & 3.98505 & 1917155 & 1917690 \\
\hline 0.223638 & 3.97409 & 3178503 & 3178696 \\
\hline 0.255507 & 3.96552 & 3867685 & 3867743 \\
\hline 0.190909 & 3.9381 & 1844497 & 1844707 \\
\hline 0.765396 & 3.90421 & 330542 & 330850 \\
\hline 0.122418 & 3.8875 & 1803765 & 1804075 \\
\hline 0.250366 & 3.88304 & 409296 & 409467 \\
\hline 0.220999 & 3.85057 & 3649246 & 3649507 \\
\hline 0.023521 & 3.81818 & 1813829 & 1813862 \\
\hline 0.0350163 & 3.81395 & 187471 & 187557 \\
\hline 0.124615 & 3.71605 & 2246626 & 2246707 \\
\hline 0.190341 & 3.69403 & 3933037 & 3933171 \\
\hline 0.276958 & 3.65574 & 2372734 & 2372978 \\
\hline 0.443216 & 3.64399 & 3411823 & 3412264 \\
\hline 0.121944 & 3.63409 & 2470851 & 2471250 \\
\hline 0.239276 & 3.57769 & 751495 & 751746 \\
\hline 0.288981 & 3.54317 & 349928 & 350206 \\
\hline 0.520376 & 3.52651 & 552399 & 553229 \\
\hline 0.758278 & 3.50655 & 3974691 & 3974920 \\
\hline 0.100441 & 3.48684 & 3853005 & 3853233 \\
\hline 0.044164 & 3.48214 & 1022293 & 1022349 \\
\hline 0.130644 & 3.45161 & 1569365 & 1569582 \\
\hline 0.154977 & 3.37956 & 4055632 & 4055769 \\
\hline 0.138281 & 3.34463 & 2784060 & 2784237 \\
\hline 0.609756 & 3.336 & 3301577 & 3302281 \\
\hline 0.33767 & 3.32574 & 376088 & 376659 \\
\hline 0.10625 & 3.29412 & 3889291 & 3889376 \\
\hline 0.31602 & 3.29109 & 3235340 & 3236938 \\
\hline 0.151261 & 3.28571 & 302935 & 303194 \\
\hline 0.22548 & 3.27803 & 1176147 & 1176370 \\
\hline 0.48913 & 3.2735 & 1064012 & 1064597 \\
\hline 0.291946 & 3.22529 & 1408781 & 1409216 \\
\hline 0.701794 & 3.17891 & 3237808 & 3238121 \\
\hline 0.20036 & 3.17365 & 3659943 & 3660281 \\
\hline 0.264211 & 3.13944 & 3422690 & 3422941 \\
\hline 0.0672727 & 3.13514 & 834804 & 834878 \\
\hline 0.356256 & 3.12046 & 1641514 & 1642203 \\
\hline 0.186887 & 3.11326 & 840326 & 840688 \\
\hline 0.41537 & 3.10539 & 2930330 & 2930757 \\
\hline 0.325564 & 3.08501 & 2078025 & 2078472 \\
\hline 0.954698 & 3.07381 & 3035865 & 3036434 \\
\hline 0.0460751 & 3 & 161464 & 161518 \\
\hline 0.13076 & 3 & 206647 & 206752 \\
\hline 0.0841674 & 3 & 656517 & 657922 \\
\hline 0.027933 & 3 & 1482263 & 1482268 \\
\hline 0.103321 & 3 & 1719819 & 1719875 \\
\hline 0.168443 & 3 & 2050380 & 2050538 \\
\hline 0.106533 & 3 & 2161523 & 2161629 \\
\hline 0.0996344 & 3 & 2664656 & 2664765 \\
\hline 0.411135 & 3 & 3006869 & 3007061 \\
\hline 0.167857 & 3 & 3440083 & 3440177 \\
\hline 0.0592532 & 3 & 3771626 & 3771699 \\
\hline 0.350413 & 3 & 572789 & 573001 \\
\hline 0.0335707 & 3 & 2193983 & 2194016 \\
\hline 0.105337 & 2.99669 & 2361188 & 2361490 \\
\hline 0.144599 & 2.98795 & 187293 & 187459 \\
\hline 0.0272126 & 2.98198 & 671439 & 671550 \\
\hline
\end{tabular}


gi_52208053_emb_BX571965 gi_52208053_emb_BX571965 gi_52208053_emb_BX571965 gi_52208053_emb_BX571965 gi_52208053_emb_BX571965 gi_52208053_emb_BX571965 gi_52208053_emb_BX571965 gi_52208053_emb_BX571965 gi_52208053_emb_BX571965 gi_52208053_emb_BX571965 gi_52208053_emb_BX571965 gi_52208053_emb_BX571965 gi_52208053_emb_BX571965 gi_52208053_emb_BX571965 gi_52208053_emb_BX571965 gi_52208053_emb_BX571965 gi_52208053_emb_BX571965 gi_52208053_emb_BX571965 gi_52208053_emb_BX571965 gi_52208053_emb_BX571965 gi_52208053_emb_BX571965 gi_52208053_emb_BX571965 gi_52208053_emb_BX571965 gi_52208053_emb_BX571965 gi_52208053_emb_BX571965 gi_52208053_emb_BX571965 gi_52208053_emb_BX571965 gi_52208053_emb_BX571965 gi_52208053_emb_BX571965 gi_52208053_emb_BX571965 gi_52208053_emb_BX571965 gi 52208053 emb_BX571965 gi_52208053_emb_BX571965 gi_52208053_emb_BX571965 gi_52208053_emb_BX571965 gi_52208053_emb_BX571965 gi_52208053_emb_BX571965 gi_52208053_emb_BX571965 gi_52208053_emb_BX571965 gi_52208053_emb_BX571965 gi_52208053_emb_BX571965 gi_52208053_emb_BX571965 gi_52208053_emb_BX571965 gi_52208053_emb_BX571965 gi_52208053_emb_BX571965 gi_52208053_emb_BX571965 gi_52208053_emb_BX571965 gi_52208053_emb_BX571965 gi 52208053 emb_BX571965 gi_52208053_emb_BX571965 gi_52208053_emb_BX571965 gi_52208053_emb_BX571965 gi_52208053_emb_BX571965 gi_52208053_emb_BX571965 gi_52208053_emb_BX571965 gi_52208053_emb_BX571965 gi_52208053_emb_BX571965 gi_52208053_emb_BX571965
19462201947071 BPSL1668 $16325201633812 \mathrm{clpX}$ 28052442806236 BPSL2323 37255473726158 BPSL3120 988966989481 BPSL0850 $1216313380 \mathrm{gspF}$ 34922083493512 BPSL2925 32260503228209 BPSL2699 22599102260410 BPSL1898 918996920036 waaF 12566351258104 BPSL1086 37910043792068 hemB 23287072330239 BPSL1957 12012211201934 hisM

167900168658 BPSL0157 23732282374277 BPSL1991 28825212883984 astD

17654221767314 kup 38161883820426 rpoC 167046167726 BPSL0156 16751121675921 BPSL1439 32083513209760 wzt

756967757530 BPSL0666 17120671713305 BPSL1476 17631841764530 purA 20589282059722 BPSL1746 19222101923109 BPSL1659 13754571376395 BPSL1191 22607712260908 BPSL1900 18567011856970 BPSL1602 $284510 \quad 285751 \mathrm{flgE}$

14941631496859 BPSL1284 19143721915463 BPSL1652 38204483824554 rpoB 31622113163431 BPSL2639 844113845339 BPSL0731 21686042168795 BPSL1821 2622326621 BPSL0024 15711071572399 BPSL1346 35193053520240 cynR 168655168801 BPSL0158 25710362572925 BPSL2142 14317681433301 BPSLr01 39514363952059 BPSL3332 29682192969418 BPSL2462 24915422493650 BPSL2077 35383963539400 araH 39762253977124 BPSL3354 23743782375523 BPSL1992 15684751569281 BPSL1344 $649836 \quad 650156$ BPSL0586 $32169 \quad 32951$ fliR 36238733626754 BPSLr05 20492232050383 BPSL1738 27735082776048 BPSL2302 39978683999388 acoD 28466702847803 BPSL2354 16545681655347 BPSL1418

\begin{tabular}{|c|c|c|c|}
\hline 0.190364 & 2.98148 & 1946566 & 1946728 \\
\hline 0.123839 & 2.96875 & 1633288 & 1633448 \\
\hline 0.363911 & 2.95845 & 2805589 & 2805950 \\
\hline 0.368249 & 2.95556 & 3725933 & 3726158 \\
\hline 0.0815534 & 2.95238 & 989191 & 989233 \\
\hline 0.0254725 & 2.93548 & 13197 & 13228 \\
\hline 0.33819 & 2.91156 & 3492648 & 3493089 \\
\hline 0.250116 & 2.90556 & 3226781 & 3227907 \\
\hline 0.3 & 2.9 & 2260260 & 2260410 \\
\hline 0.293269 & 2.89836 & 919650 & 919955 \\
\hline 0.0422056 & 2.8871 & 1257031 & 1257093 \\
\hline 0.202068 & 2.88372 & 3791180 & 3791536 \\
\hline 0.165796 & 2.87402 & 2328719 & 2328973 \\
\hline 0.102384 & 2.86301 & 1201221 & 1201294 \\
\hline 0.496042 & 2.84043 & 167900 & 168658 \\
\hline 0.0686368 & 2.83333 & 2373228 & 2373658 \\
\hline 0.120984 & 2.82486 & 2882914 & 2883091 \\
\hline 0.0882664 & 2.78443 & 1765467 & 1765634 \\
\hline 0.170363 & 2.77147 & 3816632 & 3818395 \\
\hline 0.704412 & 2.75574 & 167046 & 167726 \\
\hline 0.443758 & 2.74373 & 1675562 & 1675921 \\
\hline 0.217175 & 2.72876 & 3209393 & 3209699 \\
\hline 0.934281 & 2.72624 & 757004 & 757530 \\
\hline 0.271405 & 2.70833 & 1712967 & 1713303 \\
\hline 0.347697 & 2.69872 & 1764010 & 1764478 \\
\hline 0.574307 & 2.66886 & 2058990 & 2059446 \\
\hline 0.0767519 & 2.66667 & 1922586 & 1922655 \\
\hline 0.319829 & 2.65667 & 1376095 & 1376395 \\
\hline 1 & 2.64234 & 2260771 & 2260908 \\
\hline 0.784387 & 2.63507 & 1856706 & 1856917 \\
\hline 0.483481 & 2.62167 & 284803 & 285403 \\
\hline 0.0563798 & 2.59868 & 1496637 & 1496789 \\
\hline 0.373969 & 2.59314 & 1914448 & 1914937 \\
\hline 0.322942 & 2.5641 & 3820689 & 3823228 \\
\hline 0.34918 & 2.56338 & 3162211 & 3163413 \\
\hline 0.509788 & 2.5552 & 844639 & 845264 \\
\hline 0.183246 & 2.54286 & 2168664 & 2168699 \\
\hline 0.836683 & 2.51952 & 26288 & 26621 \\
\hline 0.128483 & 2.50602 & 1571815 & 1572369 \\
\hline 0.494118 & 2.45238 & 3519387 & 3519849 \\
\hline 1 & 2.43151 & 168655 & 168801 \\
\hline 0.140815 & 2.33083 & 2571247 & 2572333 \\
\hline 0.524462 & 2.30721 & 1432066 & 1433298 \\
\hline 0.691814 & 2.29466 & 3951436 & 3951867 \\
\hline 0.237698 & 2.29123 & 2968776 & 2969061 \\
\hline 0.127609 & 2.27881 & 2492632 & 2492901 \\
\hline 0.11753 & 2.25424 & 3539168 & 3539286 \\
\hline 0.202447 & 2.24725 & 3976456 & 3976638 \\
\hline 0.627074 & 2.22423 & 2374667 & 2375385 \\
\hline 0.141439 & 2.2193 & 1569167 & 1569281 \\
\hline 0.75 & 2.21667 & 649916 & 650156 \\
\hline 0.533248 & 2.21583 & 32471 & 32888 \\
\hline 0.492884 & 2.20493 & 3623892 & 3626602 \\
\hline 0.231034 & 2.1791 & 2050115 & 2050383 \\
\hline 0.10315 & 2.17176 & 2774038 & 2774701 \\
\hline 0.348684 & 2.1717 & 3998265 & 3998795 \\
\hline 0.149162 & 2.1716 & 2847027 & 2847196 \\
\hline 0.385109 & 2.16667 & 1655047 & 1655347 \\
\hline
\end{tabular}


gi_52208053_emb_BX571965 gi_52208053_emb_BX571965 gi_52208053_emb_BX571965 gi_52208053_emb_BX571965 gi_52208053_emb_BX571965 gi_52208053_emb_BX571965 gi_52208053_emb_BX571965 gi_52208053_emb_BX571965 gi_52208053_emb_BX571965 gi_52208053_emb_BX571965 gi_52208053_emb_BX571965 gi_52208053_emb_BX571965 gi_52208053_emb_BX571965 gi_52208053_emb_BX571965 gi_52208053_emb_BX571965 gi_52208053_emb_BX571965 gi_52208053_emb_BX571965 gi_52208053_emb_BX571965 gi_52208053_emb_BX571965 gi_52208053_emb_BX571965 gi_52208053_emb_BX571965 gi_52208053_emb_BX571965 gi_52208053_emb_BX571965 gi_52208053_emb_BX571965 gi_52208053_emb_BX571965 gi_52208053_emb_BX571965 gi_52208053_emb_BX571965 gi_52208053_emb_BX571965 gi_52208053_emb_BX571965 gi_52208053_emb_BX571965 gi_52208053_emb_BX571965 gi_52208053_emb_BX571965 gi_52208053_emb_BX571965 gi_52208053_emb_BX571965 gi_52208053_emb_BX571965 gi_52208053_emb_BX571965 gi_52208053_emb_BX571965 gi_52208053_emb_BX571965 gi_52208053_emb_BX571965 gi_52208053_emb_BX571965 gi_52208053_emb_BX571965 gi_52208053_emb_BX571965 gi_52208053_emb_BX571965 gi_52208053_emb_BX571965 gi_52208053_emb_BX571965 gi_52208053_emb_BX571965 gi_52208053_emb_BX571965 gi_52208053_emb_BX571965 gi 52208053 emb_BX571965 gi_52208053_emb_BX571965 gi_52208053_emb_BX571965 gi_52208053_emb_BX571965 gi_52208053_emb_BX571965 gi_52208053_emb_BX571965 gi_52208053_emb_BX571965 gi_52208053_emb_BX571965 gi_52208053_emb_BX571965 gi_52208053_emb_BX571965
19386311940046 BPSL1665 9441397280 BPSL0082 37458133746445 BPSL3146 39468653947629 BPSL3327 13965511396635 BPSLt07 37221173724717 BPSL3118 $26690 \quad 27412$ BPSL0025 $25193262522895 \mathrm{mfd}$ 32340273235280 BPSL2705 34988673500228 BPSL2930 $38176 \quad 39555$ BPSL0039 4216944181 BPSL0042 114323115771 BPSL0103 243932245443 BPSL0234 443964444911 BPSL0409 615702616079 BPSL0562 797270798418 BPSL0699 $864580 \quad 865065$ BPSL0749 867220868476 BPSL0751 990788991951 BPSL0852 10011251002354 BPSL0861 11023331105446 BPSL0945 11893161190755 BPSL1020 12175261218953 BPSL1048 12704301270789 BPSL1099 17920271792722 BPSL1546 18088021809608 BPSL1559 18096481811318 BPSL1560 18583411859255 BPSL1605 22444622245421 BPSL1884 27785662780665 BPSL2305 29496642950296 BPSL2445 32887203289544 BPSL2746 38928963893540 BPSL3277 39458043946868 BPSL3326 23956332398257 alaS 37901273790786 engB 29737052975111 norM 12375061239824 parC 13918491393990 pnp 35051563506427 proA 39774373979524 rep 22013282202380 BPSL1848 27859262789651 narG 31259833126945 trxB 24254382425998 BPSL2030 17928561795330 BPSL1547 $259630261258 \mathrm{dppA}$ $463292465325 \mathrm{dctB}$ $922908924101 \mathrm{pgk}$ 15020631503994 thiC 10563251059162 ileS 731943733217 BPSL0646 19899291992244 BPSL1707 19826431987463 BPSL1705 38457713846868 BPSL3239 $37044163707085 \mathrm{clpB}$ 36343183634710 paaI
$+$

$-$

$-$

$+$

$+$

$-$

$+$

$+$

$-$

$-$

$+$

$+$

$-$

\begin{tabular}{|c|c|c|c|}
\hline 0.409187 & 2.14853 & 1938655 & 1939234 \\
\hline 0.133938 & 2.14583 & 95111 & 95495 \\
\hline 0.634494 & 2.1197 & 3745855 & 3746445 \\
\hline 0.265707 & 2.09852 & 3946865 & 3947068 \\
\hline 1 & 2.09524 & 1396551 & 1396635 \\
\hline 0.0392308 & 2.08824 & 3722759 & 3722861 \\
\hline 0.621884 & 2.06682 & 26690 & 27189 \\
\hline 0.182404 & 2.04608 & 2520520 & 2522645 \\
\hline 0.243416 & 2.03934 & 3234939 & 3235280 \\
\hline 0.351947 & 2.0334 & 3499252 & 3500144 \\
\hline 0.116751 & 2 & 38785 & 38946 \\
\hline 0.0487078 & 2 & 43825 & 43923 \\
\hline 0.019337 & 2 & 114387 & 114415 \\
\hline 0.0152217 & 2 & 243932 & 243955 \\
\hline 0.0834213 & 2 & 443964 & 444043 \\
\hline 0.066313 & 2 & 615702 & 615727 \\
\hline 0.157666 & 2 & 797854 & 798035 \\
\hline 0.0824742 & 2 & 864981 & 865021 \\
\hline 0.123408 & 2 & 868104 & 868259 \\
\hline 0.0189166 & 2 & 991381 & 991403 \\
\hline 0.187144 & 2 & 1001962 & 1002192 \\
\hline 0.0848057 & 2 & 1104394 & 1104658 \\
\hline 0.0750521 & 2 & 1190647 & 1190755 \\
\hline 0.0133146 & 2 & 1217633 & 1217652 \\
\hline 0.651811 & 2 & 1270555 & 1270789 \\
\hline 0.23741 & 2 & 1792557 & 1792722 \\
\hline 0.0136476 & 2 & 1809597 & 1809608 \\
\hline 0.134132 & 2 & 1809648 & 1809872 \\
\hline 0.0634573 & 2 & 1858365 & 1858423 \\
\hline 0.0437956 & 2 & 2244803 & 2244845 \\
\hline 0.0138161 & 2 & 2780318 & 2780347 \\
\hline 0.360759 & 2 & 2949927 & 2950155 \\
\hline 0.268204 & 2 & 3288720 & 3288941 \\
\hline 0.0372671 & 2 & 3893509 & 3893533 \\
\hline 0.0516917 & 2 & 3946813 & 3946868 \\
\hline 0.0121951 & 2 & 2397697 & 2397729 \\
\hline 0.180577 & 2 & 3790127 & 3790246 \\
\hline 0.0384068 & 2 & 2974244 & 2974298 \\
\hline 0.101812 & 2 & 1238018 & 1238254 \\
\hline 0.151331 & 2 & 1391849 & 1392173 \\
\hline 0.154209 & 2 & 3505913 & 3506109 \\
\hline 0.0699569 & 2 & 3977437 & 3977583 \\
\hline 0.372624 & 1.9949 & 2201619 & 2202011 \\
\hline 0.0397315 & 1.99324 & 2787695 & 2787843 \\
\hline 0.16632 & 1.98125 & 3125994 & 3126154 \\
\hline 0.185714 & 1.98077 & 2425495 & 2425599 \\
\hline 0.0578011 & 1.97902 & 1794477 & 1794620 \\
\hline 0.369165 & 1.97504 & 259630 & 260456 \\
\hline 0.0383669 & 1.96154 & 464468 & 464546 \\
\hline 0.165968 & 1.9596 & 922922 & 923120 \\
\hline 0.215432 & 1.95673 & 1502714 & 1503130 \\
\hline 0.0398308 & 1.95575 & 1058210 & 1058323 \\
\hline 0.293564 & 1.94118 & 732798 & 733217 \\
\hline 0.133477 & 1.93204 & 1989940 & 1990249 \\
\hline 0.321162 & 1.927 & 1982643 & 1986955 \\
\hline 0.200547 & 1.89545 & 3845771 & 3845991 \\
\hline 0.302281 & 1.87925 & 3706702 & 3706921 \\
\hline 0.686224 & 1.87361 & 3634390 & 3634659 \\
\hline
\end{tabular}


gi_52208053_emb_BX571965 gi_52208053_emb_BX571965 gi_52208053_emb_BX571965 gi_52208053_emb_BX571965 gi_52208053_emb_BX571965 gi_52208053_emb_BX571965 gi_52208053_emb_BX571965 gi_52208053_emb_BX571965 gi_52208053_emb_BX571965 gi_52208053_emb_BX571965 gi_52208053_emb_BX571965 gi_52208053_emb_BX571965 gi_52208053_emb_BX571965 gi_52208053_emb_BX571965 gi_52208053_emb_BX571965 gi_52208053_emb_BX571965 gi_52208053_emb_BX571965 gi_52208053_emb_BX571965 gi_52208053_emb_BX571965 gi_52208053_emb_BX571965 gi_52208053_emb_BX571965 gi_52208053_emb_BX571965 gi_52208053_emb_BX571965 gi_52208053_emb_BX571965 gi_52208053_emb_BX571965 gi_52208053_emb_BX571965 gi_52208053_emb_BX571965 gi_52208053_emb_BX571965 gi_52208053_emb_BX571965 gi_52208053_emb_BX571965 gi_52208053_emb_BX571965 gi_52208053_emb_BX571965 gi_52208053_emb_BX571965 gi_52208053_emb_BX571965 gi_52208053_emb_BX571965 gi_52208053_emb_BX571965 gi_52208053_emb_BX571965 gi_52208053_emb_BX571965 gi_52208053_emb_BX571965 gi_52208053_emb_BX571965 gi_52208053_emb_BX571965 gi_52208053_emb_BX571965 gi_52208053_emb_BX571965 gi_52208053_emb_BX571965 gi_52208053_emb_BX571965 gi_52208053_emb_BX571965 gi_52208053_emb_BX571965 gi_52208053_emb_BX571965 gi 52208053 emb_BX571965 gi_52208053_emb_BX571965 gi_52208053_emb_BX571965 gi_52208053_emb_BX571965 gi_52208053_emb_BX571965 gi_52208053_emb_BX571965 gi_52208053_emb_BX571965 gi_52208053_emb_BX571965 gi_52208053_emb_BX571965 gi_52208053_emb_BX571965
26391172640052 BPSL2198 32763523277521 BPSL2736 15738671574889 BPSL1348 38311843834065 BPSLr08 $35164223517744 \operatorname{dctA}$ 37071193708219 BPSL3102 33023783303679 tolB 28739312875937 cyoB 38346673836200 BPSLr09 35077043508255 BPSL2937 10456181047237 BPSL0897 31307933131731 BPSL2610 104028104915 BPSL0092 912788913261 BPSL0784 13117721312755 BPSL1128 20192732021957 BPSL1715 $15831961585082 \mathrm{ftsH}$ 37702163771541 BPSL3166 12708301271198 BPSL1100 14721111474072 BPSL1270 786186786557 BPSL0689 12139011215319 otsA 22629672264232 BPSL1902 $40618114063337 \mathrm{msdA}$ 25365052537434 BPSL2111 20702862071377 cbiD 34280803429039 oxyR 718126722205 BPSL0634 947371948915 oprB 40142294015419 BPSL3384 23065192308639 BPSL1937 26289472629849 BPSL2190 14181901419416 BPSL1234 15472201549085 pckG 36273563628889 BPSLr06 10369891038575 BPSL0892a 18850851887637 BPSL1628 $35991623600358 \mathrm{ftsZ}$ 886079889639 BPSL0765 38741623876582 BPSL3258 19251481934837 BPSL1661 555370555939 BPSL0505 32331253234030 BPSL2704 18959291899177 BPSL1634 19986202017444 BPSL1712 24260512426596 BPSL2031 37465603747570 BPSL3147 33825643383121 BPSL2829 $293438 \quad 294670 \mathrm{flgL}$ 19401571944521 BPSL1666 6919670401 BPSL0062 35614453562383 BPSL2988 18728761874255 BPSL1616 34070173408243 glcF 501740503380 BPSL0465 27590552760506 BPSL2293 $108383 \quad 108727$ BPSL0097 $22853542288281 \operatorname{infB}$

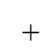

\begin{tabular}{|c|c|c|c|}
\hline 0.247059 & 1.87013 & 2639437 & 2639668 \\
\hline 0.216424 & 1.86166 & 3276734 & 3276987 \\
\hline 0.203523 & 1.85577 & 1574135 & 1574343 \\
\hline 0.619577 & 1.8549 & 3831335 & 3833581 \\
\hline 0.0907716 & 1.85 & 3517055 & 3517175 \\
\hline 0.219091 & 1.82158 & 3707491 & 3707732 \\
\hline 0.0668716 & 1.8046 & 3302406 & 3302718 \\
\hline 0.232802 & 1.79443 & 2874899 & 2875366 \\
\hline 0.547945 & 1.79405 & 3834785 & 3836195 \\
\hline 0.257713 & 1.78169 & 3507808 & 3507950 \\
\hline 0.0704138 & 1.7807 & 1046900 & 1047014 \\
\hline 0.602345 & 1.77168 & 3130794 & 3131359 \\
\hline 0.289741 & 1.74708 & 104187 & 104444 \\
\hline 0.116279 & 1.74545 & 913058 & 913113 \\
\hline 0.0508647 & 1.74 & 1312271 & 1312321 \\
\hline 0.0372578 & 1.74 & 2021857 & 2021957 \\
\hline 0.241251 & 1.73846 & 1583723 & 1584253 \\
\hline 0.146415 & 1.73711 & 3771327 & 3771541 \\
\hline 0.350543 & 1.71318 & 1270830 & 1270959 \\
\hline 0.350841 & 1.71221 & 1472794 & 1473814 \\
\hline 0.185984 & 1.71014 & 786262 & 786331 \\
\hline 0.350494 & 1.70221 & 1214610 & 1215151 \\
\hline 0.319368 & 1.69802 & 2263508 & 2264232 \\
\hline 0.136304 & 1.69712 & 4062904 & 4063112 \\
\hline 0.0947255 & 1.68182 & 2536578 & 2536666 \\
\hline 0.202566 & 1.66063 & 2070901 & 2071122 \\
\hline 0.118874 & 1.65789 & 3428145 & 3428259 \\
\hline 0.115469 & 1.64968 & 718728 & 719289 \\
\hline 0.151554 & 1.64957 & 947371 & 948292 \\
\hline 0.257983 & 1.63192 & 4014629 & 4014936 \\
\hline 0.126415 & 1.6306 & 2306983 & 2308217 \\
\hline 0.168514 & 1.625 & 2629566 & 2629718 \\
\hline 0.0668842 & 1.62195 & 1418769 & 1418851 \\
\hline 0.183914 & 1.61224 & 1547220 & 1548932 \\
\hline 0.657534 & 1.60218 & 3627399 & 3628606 \\
\hline 0.309584 & 1.58859 & 1037597 & 1038575 \\
\hline 0.106583 & 1.58088 & 1885085 & 1885357 \\
\hline 0.177258 & 1.5566 & 3599996 & 3600208 \\
\hline 0.0202247 & 1.55556 & 886600 & 887008 \\
\hline 0.287603 & 1.55172 & 3874647 & 3875578 \\
\hline 0.0603777 & 1.54701 & 1928687 & 1932941 \\
\hline 0.318102 & 1.52486 & 555468 & 555649 \\
\hline 0.474033 & 1.52448 & 3233554 & 3233983 \\
\hline 0.135468 & 1.51136 & 1898345 & 1898838 \\
\hline 0.0101466 & 1.46597 & 2012317 & 2013136 \\
\hline 0.350459 & 1.46073 & 2426051 & 2426426 \\
\hline 0.222772 & 1.45778 & 3746560 & 3746962 \\
\hline 0.0628366 & 1.45714 & 3383086 & 3383121 \\
\hline 0.353084 & 1.44368 & 293488 & 293923 \\
\hline 0.0659945 & 1.44097 & 1940357 & 1941333 \\
\hline 0.0755187 & 1.43956 & 70052 & 70143 \\
\hline 0.51919 & 1.43121 & 3561749 & 3562349 \\
\hline 0.158086 & 1.41743 & 1872876 & 1873094 \\
\hline 0.339315 & 1.41346 & 3407827 & 3408243 \\
\hline 0.0884146 & 1.4069 & 501740 & 503380 \\
\hline 0.0544452 & 1.40506 & 2759055 & 2759134 \\
\hline 0.261628 & 1.4 & 108592 & 108682 \\
\hline 0.0758456 & 1.3964 & 2286201 & 2288281 \\
\hline
\end{tabular}


gi_52208053_emb_BX571965 gi_52208053_emb_BX571965 gi_52208053_emb_BX571965 gi_52208053_emb_BX571965 gi_52208053_emb_BX571965 gi_52208053_emb_BX571965 gi_52208053_emb_BX571965 gi_52208053_emb_BX571965 gi_52208053_emb_BX571965 gi_52208053_emb_BX571965 gi_52208053_emb_BX571965 gi_52208053_emb_BX571965 gi_52208053_emb_BX571965 gi_52208053_emb_BX571965 gi_52208053_emb_BX571965 gi_52208053_emb_BX571965 gi_52208053_emb_BX571965 gi_52208053_emb_BX571965 gi_52208053_emb_BX571965 gi_52208053_emb_BX571965 gi_52208053_emb_BX571965 gi_52208053_emb_BX571965 gi_52208053_emb_BX571965 gi_52208053_emb_BX571965 gi_52208053_emb_BX571965 gi_52208053_emb_BX571965 gi_52208053_emb_BX571965 gi_52208053_emb_BX571965 gi_52208053_emb_BX571965 gi_52208053_emb_BX571965 gi_52208053_emb_BX571965 gi_52208053_emb_BX571965 gi_52208053_emb_BX571965 gi_52208053_emb_BX571965 gi_52208053_emb_BX571965 gi_52208053_emb_BX571965 gi_52208053_emb_BX571965 gi_52208053_emb_BX571965 gi_52208053_emb_BX571965 gi_52208053_emb_BX571965 gi_52208053_emb_BX571965 gi_52208053_emb_BX571965 gi_52208053_emb_BX571965 gi_52208053_emb_BX571965 gi_52208053_emb_BX571965 gi_52208053_emb_BX571965 gi_52208053_emb_BX571965 gi_52208053_emb_BX571965 gi_52208053_emb_BX571965 gi_52208053_emb_BX571965 gi_52208053_emb_BX571965 gi_52208053_emb_BX571965 gi_52208053_emb_BX571965 gi_52208053_emb_BX571965 gi_52208053_emb_BX571965 gi_52208053_emb_BX571965 gi_52208053_emb_BX571965 gi_52208053_emb_BX571965
10326161033968 BPSL0890 944167947367 bpeB $37982543799600 \mathrm{sec} Y$ 210930212273 hslU 13193001320697 BPSL1135 39896463990764 BPSL3363 12004191201198 hisP 22211162221775 BPSL1868 3378134656 BPSL0034 414152415936 argS 38775203878950 BPSL3262 13898931391170 BPSL1205 779381780688 BPSL0684 37249563725513 BPSL3119 932724933641 BPSL0804 16030531603313 BPSL1372 30309773032998 aroA\%2FtyrA 14339041436785 BPSLr02 38451043845619 BPSL3238 27896482791201 narH 10105191010854 BPSL0870 28785432879337 BPSL2383 10837661084728 BPSL0932 351992352942 BPSL0329 28013162801804 BPSL2319 2346625028 BPSL0022 40391944039634 atpF 34952613496718 BPSL2927 27072442707909 BPSL2244 18606831862650 nosZ

$1161 \quad 2375$ BPSL0002

18001141801304 BPSL1551

12817161287241 BPSL1109

26648532665980 BPSL2220

36740093675271 BPSL3080

39644553966017 BPSL3342

16553721655686 BPSL1419

$36425 \quad 37222$ BPSL0037

4056641693 BPSL0041

$57563 \quad 58372$ BPSL0053

8837490050 BPSL0078

101248102249 BPSL0089

105283106440 BPSL0093

109682110098 BPSL0099

$111591 \quad 112532$ BPSL0101

125800126804 BPSL0113A

131843134452 BPSL0118

142226144634 BPSL0127

147388150180 BPSL0130

151180151419 BPSL0133

$151423 \quad 151632$ BPSL0134

151661151858 BPSL0135

$173641 \quad 174651$ BPSL0170

233741235384 BPSL0224

273457275412 BPSL0262

290455291213 BPSL0279

305245306513 BPSL0293

316171316806 BPSL0299
0.052514

0.305

0.43685

0.210722

0.204724

0.500894

0.0179718

0.532625

0.123429

0.257287

0.146853

0.0360219

0.3443

0.254937

0.484188

0.288462

0.158337

0.635543

0.543689

0.255634

0.489552

0.331234

0.345114

0.316842

0.713115

0.492958

0.415909

0.110501

0.338346

0.0625318

0.263591

0.289076

0.0767421

0.361136

0.31775

0.103713

0.394904

0.122961

0.377107

0.306551

0.139021

0.188811

0.375108

0.0552885

0.121148

0.391434

0.0605596

0.0602159

0.00716332

0.748954

0.30622

0.177665

0.0316832

0.0663421

0.124297

0.292876

0.0449527

0.362205

\begin{tabular}{|c|c|c|}
\hline+37 & 1033278 & 10 \\
\hline 1.38832 & 944668 & \\
\hline 36224 & 3798637 & 379922 \\
\hline 36042 & 211847 & 212131 \\
\hline 36014 & 1319675 & 1319 \\
\hline 1.35893 & 3989646 & 399022 \\
\hline 1.35714 & 1201184 & 1201 \\
\hline 1.35043 & 2221286 & 222163 \\
\hline 1.32407 & 33945 & 3407 \\
\hline 1.32026 & 414277 & 41473 \\
\hline 1.31905 & 3877727 & 387793 \\
\hline 1.30435 & 1389893 & 138993 \\
\hline 1.28444 & 780160 & 780610 \\
\hline 1.28169 & 3725204 & 3725346 \\
\hline 1.27703 & 933077 & 93352 \\
\hline 1.26667 & 1603238 & 1603313 \\
\hline 1.24375 & 3031275 & 303214 \\
\hline 1.2414 & 1434272 & 1436539 \\
\hline 1.23214 & 3845339 & $384561 \mathrm{C}$ \\
\hline 1.22922 & 2789916 & 279113 \\
\hline 1.21951 & 1010519 & 101068 \\
\hline 1.19772 & 2879074 & 2879337 \\
\hline 1.18976 & 1084387 & $108471 \mathrm{C}$ \\
\hline 1.1794 & 351992 & 35229 \\
\hline 1.16954 & 2801316 & 280166 \\
\hline 1.16494 & 23976 & 2478 \\
\hline 1.16393 & 4039414 & 4039597 \\
\hline 1.16149 & 3495369 & 3495530 \\
\hline 1.15111 & 2707684 & 2707909 \\
\hline 1.14634 & 1862527 & 1862650 \\
\hline 1.14375 & 1602 & 1922 \\
\hline 1.09012 & 1800395 & 1800816 \\
\hline 1.08019 & 1282825 & 1285743 \\
\hline 1.05651 & 2665231 & 2665763 \\
\hline 1.02743 & 3674862 & 3675263 \\
\hline 1.01852 & 3965855 & 3966017 \\
\hline 1.00806 & 1655372 & 165549 \\
\hline 1 & 36898 & 3699 \\
\hline 1 & 40923 & 4134 \\
\hline 1 & 58041 & 5828 \\
\hline 1 & 89099 & 89332 \\
\hline 1 & 101277 & 10146 \\
\hline 1 & 105294 & 10572 \\
\hline 1 & 109682 & 10970 \\
\hline 1 & 112142 & 11225 \\
\hline 1 & 126061 & 12651 \\
\hline 1 & 132173 & 13233 \\
\hline 1 & 143150 & 14329 \\
\hline 1 & 148574 & 14859 \\
\hline 1 & 151180 & 15135 \\
\hline 1 & 151568 & 15163 \\
\hline 1 & 151661 & 15169 \\
\hline 1 & 174097 & 17412 \\
\hline 1 & 234870 & 23497 \\
\hline 1 & 274584 & 27482 \\
\hline 1 & 290879 & 29110 \\
\hline 1 & 306369 & 30642 \\
\hline 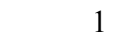 & 316319 & 31654 \\
\hline
\end{tabular}


gi_52208053_emb_BX571965 gi_52208053_emb_BX571965 gi_52208053_emb_BX571965 gi_52208053_emb_BX571965 gi_52208053_emb_BX571965 gi_52208053_emb_BX571965 gi_52208053_emb_BX571965 gi_52208053_emb_BX571965 gi_52208053_emb_BX571965 gi_52208053_emb_BX571965 gi_52208053_emb_BX571965 gi_52208053_emb_BX571965 gi_52208053_emb_BX571965 gi_52208053_emb_BX571965 gi_52208053_emb_BX571965 gi_52208053_emb_BX571965 gi_52208053_emb_BX571965 gi_52208053_emb_BX571965 gi_52208053_emb_BX571965 gi_52208053_emb_BX571965 gi_52208053_emb_BX571965 gi_52208053_emb_BX571965 gi_52208053_emb_BX571965 gi_52208053_emb_BX571965 gi_52208053_emb_BX571965 gi_52208053_emb_BX571965 gi_52208053_emb_BX571965 gi_52208053_emb_BX571965 gi_52208053_emb_BX571965 gi_52208053_emb_BX571965 gi_52208053_emb_BX571965 gi 52208053 emb_BX571965 gi_52208053_emb_BX571965 gi_52208053_emb_BX571965 gi_52208053_emb_BX571965 gi_52208053_emb_BX571965 gi_52208053_emb_BX571965 gi_52208053_emb_BX571965 gi_52208053_emb_BX571965 gi_52208053_emb_BX571965 gi_52208053_emb_BX571965 gi_52208053_emb_BX571965 gi_52208053_emb_BX571965 gi_52208053_emb_BX571965 gi_52208053_emb_BX571965 gi_52208053_emb_BX571965 gi_52208053_emb_BX571965 gi_52208053_emb_BX571965 gi 52208053 emb_BX571965 gi_52208053_emb_BX571965 gi_52208053_emb_BX571965 gi_52208053_emb_BX571965 gi_52208053_emb_BX571965 gi_52208053_emb_BX571965 gi_52208053_emb_BX571965 gi_52208053_emb_BX571965 gi_52208053_emb_BX571965 gi_52208053_emb_BX571965
339593340132 BPSL0319

362767363495 BPSL0341

365609368338 BPSL0345

389216390619 BPSL0361

397561398844 BPSL0368

413225414073 BPSL0382

430475431254 BPSL0397

448619448993 BPSL0415

489595490560 BPSL0451

516794518146 BPSL0476

581037581669 BPSL0528

589316590299 BPSL0538

$617396 \quad 621349$ BPSL0565

631502633238 BPSL0574

673053675353 BPSL0600

$698536 \quad 699402$ BPSL0617

699399700817 BPSL0618

707731709326 BPSL0625

734064735851 BPSL0648

740796741620 BPSL0652

762676763563 BPSL0670

816038817771 BPSL0713

819482820171 BPSL0716

830934832055 BPSL0723

845513847321 BPSL0732

$851785 \quad 852705$ BPSL0736

863547864605 BPSL0748

868473869510 BPSL0752

874119874562 BPSL0757

$877250 \quad 877804$ BPSL0762

878619881801 BPSL0763

896323896559 BPSL0771

896537898423 BPSL0772

902391903134 BPSL0775

931480932619 BPSL0803

939851940273 BPSL0809

959899960675 BPSL0826

961624963120 BPSL0828

963171964499 BPSL0829

982322983254 BPSL0844

10355991036444 BPSL0892

10733431074293 BPSL0920

10859481086844 BPSL0934

10932131097322 BPSL0941

11219141123011 BPSL0964

11336781135735 BPSL0976

11660381168317 BPSL1003

11773091178124 BPSL1011

12693541270196 BPSL1098

12874131287934 BPSL1110

13105071311775 BPSL1127

13863301387877 BPSL1202

14299401431157 BPSL1246

14515941452886 BPSL1257

14809681482023 BPSL1278

14870131490357 BPSL1282

14903541494166 BPSL1283

15070981507889 BPSL1294
$-$

$+$

$-$

$+$

$-$

-

$-$

$-$

$+$

-

$-$

$-$

$-$

$+$

$+$

$+$

$+$

$+$

$+$

-

-

$-$

$+$

$-$

$-$

-

$+$

$+$

$+$

$+$

$+$

-

$-$

$-$

$-$

$+$

$+$

$+$

$+$

$+$

+
+

$+$

$+$

$+$

$-$

$+$

$+$

-

$-$

$-$

-

-

$+$

-

\begin{tabular}{|c|c|c|c|}
\hline 0.198516 & 1 & 339593 & 339700 \\
\hline 0.225275 & 1 & 362913 & 363077 \\
\hline 0.1048 & 1 & 366493 & 368304 \\
\hline 0.27655 & 1 & 390166 & 390554 \\
\hline 0.13562 & 1 & 397561 & 397735 \\
\hline 0.183962 & 1 & 413866 & 414022 \\
\hline 0.0487805 & 1 & 431138 & 431176 \\
\hline 0.352941 & 1 & 448723 & 448855 \\
\hline 0.150259 & 1 & 489986 & 490131 \\
\hline 0.0769231 & 1 & 517307 & 517411 \\
\hline 0.327532 & 1 & 581279 & 581486 \\
\hline 0.0528993 & 1 & 589733 & 589785 \\
\hline 0.0574247 & 1 & 618767 & 618994 \\
\hline 0.0408986 & 1 & 632715 & 632786 \\
\hline 0.0256522 & 1 & 673053 & 673112 \\
\hline 0.136259 & 1 & 699284 & 699402 \\
\hline 0.0197461 & 1 & 699399 & 699427 \\
\hline 0.0219436 & 1 & 708288 & 708323 \\
\hline 0.0509233 & 1 & 735354 & 735445 \\
\hline 0.00364078 & 1 & 741617 & 741620 \\
\hline 0.120631 & 1 & 762814 & 762921 \\
\hline 0.130987 & 1 & 817155 & 817382 \\
\hline 0.380261 & 1 & 819892 & 820154 \\
\hline 0.0160571 & 1 & 831095 & 831113 \\
\hline 0.220686 & 1 & 845971 & 846370 \\
\hline 0.0836957 & 1 & 851785 & 851862 \\
\hline 0.340265 & 1 & 863609 & 863969 \\
\hline 0.22758 & 1 & 868823 & 869059 \\
\hline 0.255079 & 1 & 874119 & 874232 \\
\hline 0.0703971 & 1 & 877306 & 877345 \\
\hline 0.0370836 & 1 & 881439 & 881557 \\
\hline 0.105932 & 1 & 896323 & 896348 \\
\hline 0.0164369 & 1 & 896722 & 896753 \\
\hline 0.131898 & 1 & 903036 & 903134 \\
\hline 0.181738 & 1 & 932307 & 932514 \\
\hline 0.218009 & 1 & 939919 & 940011 \\
\hline 0.0811856 & 1 & 960612 & 960675 \\
\hline 0.118316 & 1 & 962636 & 963120 \\
\hline 0.0933735 & 1 & 963171 & 963295 \\
\hline 0.256438 & 1 & 983015 & 983254 \\
\hline 0.151479 & 1 & 1035769 & 1035897 \\
\hline 0.183158 & 1 & 1073465 & 1073639 \\
\hline 0.0256696 & 1 & 1085989 & 1086012 \\
\hline 0.0316379 & 1 & 1097156 & 1097286 \\
\hline 0.0309936 & 1 & 1122166 & 1122200 \\
\hline 0.0330579 & 1 & 1134921 & 1134989 \\
\hline 0.0776656 & 1 & 1166052 & 1166229 \\
\hline 0.0957055 & 1 & 1178046 & 1178124 \\
\hline 0.109264 & 1 & 1270104 & 1270196 \\
\hline 0.00191939 & 1 & 1287933 & 1287934 \\
\hline 0.035489 & 1 & 1311488 & 1311533 \\
\hline 0.147382 & 1 & 1387256 & 1387484 \\
\hline 0.0714873 & 1 & 1430858 & 1430945 \\
\hline 0.522446 & 1 & 1451711 & 1452437 \\
\hline 0.418009 & 1 & 1481303 & 1481744 \\
\hline 0.0191388 & 1 & 1488642 & 1488706 \\
\hline 0.00629591 & 1 & 1492810 & 1492834 \\
\hline 0.0366625 & 1 & 1507860 & 1507889 \\
\hline
\end{tabular}


gi_52208053_emb_BX571965 gi_52208053_emb_BX571965 gi_52208053_emb_BX571965 gi_52208053_emb_BX571965 gi_52208053_emb_BX571965 gi_52208053_emb_BX571965 gi_52208053_emb_BX571965 gi_52208053_emb_BX571965 gi_52208053_emb_BX571965 gi_52208053_emb_BX571965 gi_52208053_emb_BX571965 gi_52208053_emb_BX571965 gi_52208053_emb_BX571965 gi_52208053_emb_BX571965 gi_52208053_emb_BX571965 gi_52208053_emb_BX571965 gi_52208053_emb_BX571965 gi_52208053_emb_BX571965 gi_52208053_emb_BX571965 gi_52208053_emb_BX571965 gi_52208053_emb_BX571965 gi_52208053_emb_BX571965 gi_52208053_emb_BX571965 gi_52208053_emb_BX571965 gi_52208053_emb_BX571965 gi_52208053_emb_BX571965 gi_52208053_emb_BX571965 gi_52208053_emb_BX571965 gi_52208053_emb_BX571965 gi_52208053_emb_BX571965 gi_52208053_emb_BX571965 gi_52208053_emb_BX571965 gi_52208053_emb_BX571965 gi_52208053_emb_BX571965 gi_52208053_emb_BX571965 gi_52208053_emb_BX571965 gi_52208053_emb_BX571965 gi_52208053_emb_BX571965 gi_52208053_emb_BX571965 gi_52208053_emb_BX571965 gi_52208053_emb_BX571965 gi_52208053_emb_BX571965 gi_52208053_emb_BX571965 gi_52208053_emb_BX571965 gi_52208053_emb_BX571965 gi_52208053_emb_BX571965 gi_52208053_emb_BX571965 gi_52208053_emb_BX571965 gi 52208053 emb_BX571965 gi_52208053_emb_BX571965 gi_52208053_emb_BX571965 gi_52208053_emb_BX571965 gi_52208053_emb_BX571965 gi_52208053_emb_BX571965 gi_52208053_emb_BX571965 gi_52208053_emb_BX571965 gi_52208053_emb_BX571965 gi_52208053_emb_BX571965
15128621513689 BPSL1299 15172031518027 BPSL1302 15181291519190 BPSL1303 15816181582172 BPSL1354 16033101603720 BPSL1373 16108491611562 BPSL1381 16567941658371 BPSL1422 16603791662463 BPSL1424 16717931672812 BPSL1435 17067951707802 BPSL1471 17078091708960 BPSL1472 17276661729504 BPSL1490 17735841774408 BPSL1531 17899021790447 BPSL1544 18197251822895 BPSL1567 18457971846417 BPSL1592 18464571846575 BPSL1592A 18575081858344 BPSL1604 18687381870450 BPSL1613 18719071872869 BPSL1615 18742961875738 BPSL1617 18897711893145 BPSL1631 19039621904729 BPSL1639 19445181946188 BPSL1667 19503451950824 BPSL1671 19550291956663 BPSL1676 19968081998529 BPSL1711 20237722025004 BPSL1718 20273692028583 BPSL1721 20482282049166 BPSL1737 21219062122796 BPSL1784 21282822129373 BPSL1790 21321892133133 BPSL1793 21332382134254 BPSL1794 21703162171155 BPSL1823 21806712182575 BPSL1829 22026672203113 BPSL1849 22032862203762 BPSL1850 22140222215164 BPSL1860 22365532238265 BPSL1878 22426302244402 BPSL1883 22570782258403 BPSL1895 22941252295027 BPSL1923 22979502298228 BPSL1929 22986842298974 BPSL1931 23193862319739 BPSL1947 23231362323693 BPSL1951 23237082324808 BPSL1952 23406412341945 BPSL1966 23431492344570 BPSL1969 23665902367936 BPSL1984 23882112388546 BPSL2003 23987922400012 BPSL2010 24003412400547 BPSL2011 24040722406120 BPSL2015 24174182418038 BPSL2024 24205432421508 BPSL2026 24215242423872 BPSL2027

\begin{tabular}{|c|}
\hline \\
\hline $\begin{array}{r}0.0918984 \\
0.174757\end{array}$ \\
\hline 0.0471254 \\
\hline 0.0415162 \\
\hline 0.943902 \\
\hline 0.215989 \\
\hline 0.0469245 \\
\hline 0.0230326 \\
\hline 0.0235525 \\
\hline 0.163853 \\
\hline 0.329279 \\
\hline 0.0136017 \\
\hline 0.144417 \\
\hline 0.188991 \\
\hline 0.0526814 \\
\hline 0.146774 \\
\hline 0.516949 \\
\hline 0.113636 \\
\hline 0.0905374 \\
\hline 0.0135135 \\
\hline 0.0416089 \\
\hline 0.0509781 \\
\hline 0.0625815 \\
\hline 0.15509 \\
\hline 0.427975 \\
\hline 0.0893513 \\
\hline 0.138873 \\
\hline 0.012987 \\
\hline 0.0140033 \\
\hline 0.228145 \\
\hline 0.111236 \\
\hline 0.0531622 \\
\hline 0.0286017 \\
\hline 0.0177165 \\
\hline 0.0417163 \\
\hline 0.075105 \\
\hline 0.392377 \\
\hline 0.136555 \\
\hline 0.11296 \\
\hline 0.0712617 \\
\hline 0.0124153 \\
\hline 0.173585 \\
\hline 0.371397 \\
\hline 0.672662 \\
\hline 0.158621 \\
\hline 0.0679887 \\
\hline 0.40395 \\
\hline 0.113636 \\
\hline 0.312117 \\
\hline 0.0309641 \\
\hline 0.137444 \\
\hline 0.146269 \\
\hline 0.345902 \\
\hline 0.0339806 \\
\hline 0.0537109 \\
\hline 0.269355 \\
\hline 0.290155 \\
\hline 0.016184 \\
\hline
\end{tabular}

$\begin{array}{ll}1513333 & 1513409\end{array}$ $\begin{array}{ll}1517562 & 1517706\end{array}$ $1518743 \quad 1518793$ $\begin{array}{ll}1581917 \quad 1581940 \\ 1603310 & 1603697\end{array}$ $\begin{array}{ll}1603310 & 1603697\end{array}$ $1611408 \quad 1611562$ $1656879 \quad 1656953$ $\begin{array}{ll}1660540 & 1660588\end{array}$ $\begin{array}{ll}1671953 & 1671977\end{array}$ $\begin{array}{ll}1707502 & 1707667\end{array}$ $\begin{array}{ll}1708560 & 1708939\end{array}$ $\begin{array}{ll}1728832 & 1728857\end{array}$ $1774143 \quad 1774262$ $1790143 \quad 1790246$ $\begin{array}{ll}1820212 & 1820379\end{array}$ $\begin{array}{ll}1846326 & 1846417\end{array}$ $\begin{array}{ll}1846457 & 1846518\end{array}$ $\begin{array}{ll}1857928 & 1858023\end{array}$ $\begin{array}{ll}1868738 & 1868893\end{array}$ $\begin{array}{ll}1872856 & 1872869\end{array}$ $\begin{array}{ll}1875186 & 1875246\end{array}$ $1892021 \quad 1892193$ $1904456 \quad 1904504$ $1945853 \quad 1946173$ $1950345 \quad 1950550$ $1956294 \quad 1956440$ $1997735 \quad 1997974$ $2024988 \quad 2025004$ $2028116 \quad 2028133$ $\begin{array}{ll}2048415 & 2048629\end{array}$ $2122250 \quad 2122349$ $2129210 \quad 2129268$ 21329292132956 $2133238 \quad 2133256$ 21709332170968

$1 \quad 2180671 \quad 2180814$

$1 \quad 2202843 \quad 2203113$

$1 \quad 2203428 \quad 2203493$

$1 \quad 2214321 \quad 2214450$

$1 \quad 2237418 \quad 2237540$

$1 \quad 2243542 \quad 2243564$

$12257489 \quad 2258109$

$1 \quad 2294623 \quad 2294958$

$1 \quad 2297950 \quad 2298137$

$\begin{array}{lll}1 & 2298928 & 2298974\end{array}$

$1 \quad 2319629 \quad 2319653$

$1 \quad 2323136 \quad 2323361$

$1 \quad 2323708 \quad 2323833$

$1 \quad 2341504 \quad 2341911$

$1 \quad 2343515 \quad 2343559$

$1 \quad 2367154 \quad 2367339$

$12388443 \quad 2388492$

$12399103 \quad 2400006$

$1 \quad 2400540 \quad 2400547$

$1 \quad 2405850 \quad 2405960$

$1 \quad 2417418 \quad 2417585$

$12420615 \quad 2421508$

$1 \quad 2421524 \quad 2421562$ 
gi_52208053_emb_BX571965 gi_52208053_emb_BX571965 gi_52208053_emb_BX571965 gi_52208053_emb_BX571965 gi_52208053_emb_BX571965 gi_52208053_emb_BX571965 gi_52208053_emb_BX571965 gi_52208053_emb_BX571965 gi_52208053_emb_BX571965 gi_52208053_emb_BX571965 gi_52208053_emb_BX571965 gi_52208053_emb_BX571965 gi_52208053_emb_BX571965 gi_52208053_emb_BX571965 gi 52208053 emb_BX571965 gi_52208053_emb_BX571965 gi_52208053_emb_BX571965 gi_52208053_emb_BX571965 gi_52208053_emb_BX571965 gi_52208053_emb_BX571965 gi_52208053_emb_BX571965 gi_52208053_emb_BX571965 gi_52208053_emb_BX571965 gi_52208053_emb_BX571965 gi_52208053_emb_BX571965 gi_52208053_emb_BX571965 gi_52208053_emb_BX571965 gi_52208053_emb_BX571965 gi_52208053_emb_BX571965 gi_52208053_emb_BX571965 gi_52208053_emb_BX571965 gi 52208053 emb_BX571965 gi_52208053_emb_BX571965 gi_52208053_emb_BX571965 gi_52208053_emb_BX571965 gi_52208053_emb_BX571965 gi_52208053_emb_BX571965 gi_52208053_emb_BX571965 gi_52208053_emb_BX571965 gi_52208053_emb_BX571965 gi_52208053_emb_BX571965 gi_52208053_emb_BX571965 gi_52208053_emb_BX571965 gi_52208053_emb_BX571965 gi_52208053_emb_BX571965 gi_52208053_emb_BX571965 gi_52208053_emb_BX571965 gi_52208053_emb_BX571965 gi 52208053 emb_BX571965 gi_52208053_emb_BX571965 gi_52208053_emb_BX571965 gi_52208053_emb_BX571965 gi_52208053_emb_BX571965 gi_52208053_emb_BX571965 gi_52208053_emb_BX571965 gi_52208053_emb_BX571965 gi_52208053_emb_BX571965 gi_52208053_emb_BX571965
24239802424819 BPSL2028 24385582441398 BPSL2041 24469792449387 BPSL2047 24548232455089 BPSL2051 24550862456528 BPSL2052 25106362511892 BPSL2091 25625712563539 BPSL2134 26341152634978 BPSL2195 26450292646981 BPSL2203 26481462648499 BPSL2205 26492242651545 BPSL2207 26680972668936 BPSL2223 26717402672618 BPSL2227 26726232673420 BPSL2228 26734172682440 BPSL2229 26902942691574 BPSL2234 27190912719636 BPSL2254 27197292720892 BPSL2255 27252142726416 BPSL2260 27316042732554 BPSL2267 27326382732985 BPSL2268 27457832747477 BPSL2279 27586842758944 BPSL2292 27624952763958 BPSL2296 27760452776683 BPSL2303 28020572802875 BPSL2320 28139062815288 BPSL2328 28377962838521 BPSL2347 28770182878250 BPSL2382 29648592965848 BPSL2459 30412113041936 BPSL2524 30830263083502 BPSL2554 31299463130803 BPSL2609 31738593175166 BPSL2651 31870943187978 BPSL2664 31929433194100 BPSL2669 31941153195137 BPSL2670 31951653196271 BPSL2671 32299503230957 BPSL2701 32436153245057 BPSL2713 32730063273986 BPSL2733 32755773276236 BPSL2735 33640333364776 BPSL2813 35298013530232 BPSL2958 35342073534680 BPSL2961 35411153542110 BPSL2968 35464273548061 BPSL2974 35545613555217 BPSL2980 35593643560773 BPSL2986 35773953578858 BPSL3001 36169643617590 BPSL3035 36715843673185 BPSL3078 36853433687508 BPSL3089 36949483695934 BPSL3095 36971903701083 BPSL3097 37184343719234 BPSL3114 37537633755529 BPSL3155 37746183775946 BPSL3170
$-$

$-$

$-$

$+$

$+$

$-$

$+$

$+$

$-$

$-$

$-$

$-$

$-$

$-$

-

$+$

$-$

$+$

-

$+$

$+$

$+$

$-$

$-$

$+$

-

-

$-$

$+$

-

$+$

$-$

$-$

$+$

$-$

$+$

$+$

$+$

$+$

$+$

$+$

$+$

-

$-$

$+$

-

$-$

$+$

$+$

-

$-$

$-$

$+$

-

$+$

$+$

$+$

$-$

\begin{tabular}{rrrr}
0.27056 & 1 & 2424536 & 2424763 \\
0.00809859 & 1 & 2439356 & 2439379 \\
0.129983 & 1 & 2447600 & 2447913 \\
1 & 1 & 2454823 & 2455089 \\
0.0270458 & 1 & 2455086 & 2455125 \\
0.013535 & 1 & 2510946 & 2510963 \\
0.183884 & 1 & 2562617 & 2562795 \\
0.0926999 & 1 & 2634802 & 2634882 \\
0.0384221 & 1 & 2645220 & 2645295 \\
0.0934844 & 1 & 2648466 & 2648499 \\
0.093925 & 1 & 2651062 & 2651280 \\
0.153754 & 1 & 2668597 & 2668726 \\
0.0512528 & 1 & 2672573 & 2672618 \\
0.0840652 & 1 & 2672623 & 2672690 \\
0.0115261 & 1 & 2674332 & 2674436 \\
0.195312 & 1 & 2690805 & 2691055 \\
0.8 & 1 & 2719189 & 2719625 \\
0.143594 & 1 & 2719729 & 2720892 \\
0.014975 & 1 & 2725269 & 2725287 \\
0.0157895 & 1 & 2732539 & 2732554 \\
0.00288184 & 1 & 2732638 & 2732639 \\
0.152302 & 1 & 2747045 & 2747303 \\
0.507692 & 1 & 2758812 & 2758944 \\
0.159945 & 1 & 2763172 & 2763406 \\
0.0548589 & 1 & 2776504 & 2776539 \\
0.179707 & 1 & 2802510 & 2802657 \\
0.0759768 & 1 & 2815106 & 2815211 \\
0.475862 & 1 & 2837821 & 2838166 \\
0.201299 & 1 & 2877565 & 2877813 \\
0.0586451 & 1 & 2965144 & 2965202 \\
0.097931 & 1 & 3041672 & 3041743 \\
0.140756 & 1 & 3083435 & 3083502 \\
0.0105018 & 1 & 3130794 & 3130803 \\
0.0596787 & 1 & 3174664 & 3174742 \\
0.222851 & 1 & 3187619 & 3187816 \\
0.0302506 & 1 & 3193924 & 3193959 \\
0.192759 & 1 & 3194837 & 3195137 \\
0.299277 & 1 & 3195165 & 3195496 \\
0.0397219 & 1 & 3230222 & 3230262 \\
0.090846 & 1 & 3243897 & 3244455 \\
0.0367347 & 1 & 3273374 & 3273410 \\
0.0986343 & 1 & 3276027 & 3276092 \\
0.0309556 & 1 & 3364647 & 3364670 \\
0.206497 & 1 & 3530117 & 3530206 \\
0.473573 & 1 & 3534207 & 3534431 \\
0.0683417 & 1 & 3542042 & 3542110 \\
0.0954712 & 1 & 3547905 & 3548061 \\
0.306402 & 1 & 3554675 & 3554876 \\
0.0141945 & 1 & 3559515 & 3559535 \\
0.256323 & 1 & 3578102 & 3578477 \\
0.0527157 & 1 & 3617557 & 3617590 \\
0.0349781 & 1 & 3671921 & 3671977 \\
0.08711359 & 1 & 3685343 & 3685362 \\
0.0662728 & 1 & 3695501 & 3695934 \\
0.06875 & 1 & 3699389 & 3699647 \\
0.0198188 & 1 & 3718484 & 3718539 \\
0.0850904 & 1 & 3753763 & 3753798 \\
& 1 & 3774698 & 3774811 \\
\hline
\end{tabular}


gi_52208053_emb_BX571965 gi_52208053_emb_BX571965 gi_52208053_emb_BX571965 gi_52208053_emb_BX571965 gi_52208053_emb_BX571965 gi_52208053_emb_BX571965 gi_52208053_emb_BX571965 gi_52208053_emb_BX571965 gi_52208053_emb_BX571965 gi_52208053_emb_BX571965 gi_52208053_emb_BX571965 gi_52208053_emb_BX571965 gi_52208053_emb_BX571965 gi_52208053_emb_BX571965 gi_52208053_emb_BX571965 gi_52208053_emb_BX571965 gi_52208053_emb_BX571965 gi_52208053_emb_BX571965 gi_52208053_emb_BX571965 gi_52208053_emb_BX571965 gi_52208053_emb_BX571965 gi_52208053_emb_BX571965 gi_52208053_emb_BX571965 gi_52208053_emb_BX571965 gi_52208053_emb_BX571965 gi_52208053_emb_BX571965 gi_52208053_emb_BX571965 gi_52208053_emb_BX571965 gi_52208053_emb_BX571965 gi_52208053_emb_BX571965 gi_52208053_emb_BX571965 gi 52208053 emb_BX571965 gi_52208053_emb_BX571965 gi_52208053_emb_BX571965 gi_52208053_emb_BX571965 gi_52208053_emb_BX571965 gi_52208053_emb_BX571965 gi_52208053_emb_BX571965 gi_52208053_emb_BX571965 gi_52208053_emb_BX571965 gi_52208053_emb_BX571965 gi_52208053_emb_BX571965 gi_52208053_emb_BX571965 gi_52208053_emb_BX571965 gi_52208053_emb_BX571965 gi_52208053_emb_BX571965 gi_52208053_emb_BX571965 gi_52208053_emb_BX571965 gi 52208053 emb_BX571965 gi_52208053_emb_BX571965 gi_52208053_emb_BX571965 gi_52208053_emb_BX571965 gi_52208053_emb_BX571965 gi_52208053_emb_BX571965 gi_52208053_emb_BX571965 gi_52208053_emb_BX571965 gi_52208053_emb_BX571965 gi_52208053_emb_BX571965
38476213851202 BPSL3241 38712313872637 BPSL3256 38840373884951 BPSL3268 38916603892295 BPSL3276 38994193900561 BPSL3284 40320114033741 BPSL3394 $127411 \quad 127486$ BPSLt01 26368892636964 BPSLt25 38300133830098 BPSLt48 31797633180761 UreD $21478182150949 \mathrm{amrB}$ 40370644038605 atpA 395651396997 bioA 393751394473 bioD 941844942479 bpeR $230375 \quad 231793 \mathrm{calB}$ $21889112189837 \mathrm{cbl}$ $16317271632380 \mathrm{clpP}$ 20684912069216 cobM 20840412087886 cobN $492944 \quad 494551 \mathrm{ctaD}$ 27236532724555 dapA $12957591299283 \mathrm{dnaE}$ 509901513119 dnaE2 30135283014277 etfB 40007644002161 eutB $30747113075727 \mathrm{fbp}$ 30471053050059 fdsA 283631284482 flgD 291419293422 flgK 39113563913458 flhA $239562 \quad 240557 \mathrm{fliG}$ $237324 \quad 238895$ fliI $26520502652823 \mathrm{fpr}$ $36130153614859 \mathrm{ftsI}$ $24892732491489 \mathrm{glgB}$ $15861111587469 \mathrm{glmM}$ $333510 \quad 334871 \mathrm{glmU}$ 27982112799305 glnL 34910823491984 gltI 26371352638544 gltX 759664760671 glyQ 33421683342761 gmhA 483930484679 gpmA 37398533741190 hisD 12028311203607 hisJ 17619111763059 hisZ $32807223282074 \mathrm{hmgA}$ $27546612755188 \mathrm{hscB}$ $12582401260138 \mathrm{htpG}$ 28300502831738 hutU 39936263995329 ilvG 23141632314699 infC 17515691752819 ispG 13846481386195 leuA 16340081636425 lon 27490352750561 lysS 13391131339742 maf
0.0597598

0.0369844

0.135667

0.411024

0.279335

0.269364

0.533333

0.4

0.388235

0.258517

0.0795273

0.211551

0.156018

0.139889

0.286614

0.0148096

0.087473

0.271057

0.205517

0.0470741

0.100187

0.190687

0.034336

0.00994406

0.0387183

0.107373

0.139764

0.0179418

0.0258519

0.158263

0.0466223

0.182915

0.359007

0.644243

0.235358

0.00812274

0.122975

0.0382072

0.0566728

0.564302

0.100071

0.353525

0.06914

0.156208

0.0897532

0.148196

0.043554

0.0954142

0.12334

0.0200211

0.0242891

0.0669407

0.0652985

0.1984

0.0174531

0.0678527

0.0334207

0.265501
$3848022 \quad 3848236$

$3872585 \quad 3872637$

$3884742 \quad 3884866$

$3892034 \quad 3892295$

$3900070 \quad 3900389$

$4032542 \quad 4033008$

$127446 \quad 127486$

$2636900 \quad 2636930$

$3830055 \quad 3830088$

$3180329 \quad 3180587$

$2147973 \quad 2148222$

$4037703 \quad 4038029$

$396163 \quad 396373$

$394237 \quad 394338$

942237942419

$230969 \quad 230990$

$2188993 \quad 2189074$

$1632052 \quad 1632229$

$2068497 \quad 2069174$

$2084222 \quad 2084403$

$493800 \quad 493961$

$2723911 \quad 2724083$

$1296327 \quad 1297895$

$510371 \quad 510403$

$3014248 \quad 3014277$

$4001640 \quad 4001790$

$3075485 \quad 3075627$

$3049183 \quad 3049236$

$283698 \quad 283720$

$293017 \quad 293334$

39129123913010

$240001 \quad 240183$

$237611 \quad 238175$

$2652057 \quad 2652653$

$3614149 \quad 3614583$

$2490034 \quad 2490052$

$1586815 \quad 1586982$

$333820 \quad 333872$

$2798399 \quad 2798461$

$3491350 \quad 3491859$

$2637634 \quad 2637775$

$760126 \quad 760482$

$3342513 \quad 3342554$

$483930 \quad 484568$

$3740596 \quad 3740716$

$1203292 \quad 1203407$

$1762864 \quad 1762914$

$3280799 \quad 3280928$

$2755096 \quad 2755161$

$1259313 \quad 1259351$

$2831180 \quad 2831221$

$3994993 \quad 3995107$

$2314585 \quad 2314620$

$1752148 \quad 1752396$

$1385928 \quad 1385955$

$1635395 \quad 1635559$

$2749702 \quad 2749753$

$1339353 \quad 1339520$ 
gi_52208053_emb_BX571965 gi_52208053_emb_BX571965 gi_52208053_emb_BX571965 gi_52208053_emb_BX571965 gi_52208053_emb_BX571965 gi_52208053_emb_BX571965 gi_52208053_emb_BX571965 gi_52208053_emb_BX571965 gi_52208053_emb_BX571965 gi_52208053_emb_BX571965 gi_52208053_emb_BX571965 gi_52208053_emb_BX571965 gi_52208053_emb_BX571965 gi_52208053_emb_BX571965 gi 52208053 emb_BX571965 gi_52208053_emb_BX571965 gi_52208053_emb_BX571965 gi_52208053_emb_BX571965 gi_52208053_emb_BX571965 gi_52208053_emb_BX571965 gi_52208053_emb_BX571965 gi_52208053_emb_BX571965 gi_52208053_emb_BX571965 gi_52208053_emb_BX571965 gi_52208053_emb_BX571965 gi_52208053_emb_BX571965 gi_52208053_emb_BX571965 gi_52208053_emb_BX571965 gi_52208053_emb_BX571965 gi_52208053_emb_BX571965 gi_52208053_emb_BX571965 gi 52208053 emb_BX571965 gi_52208053_emb_BX571965 gi_52208053_emb_BX571965 gi_52208053_emb_BX571965 gi_52208053_emb_BX571965 gi_52208053_emb_BX571965 gi_52208053_emb_BX571965 gi_52208053_emb_BX571965 gi_52208053_emb_BX571965 gi_52208053_emb_BX571965 gi_52208053_emb_BX571965 gi_52208053_emb_BX571965 gi_52208053_emb_BX571965 gi_52208053_emb_BX571965
11598151161992 metG 419469420548 metH2 $30160153017049 \operatorname{metN}$ $767263768636 \mathrm{miaB}$ $29591792960261 \mathrm{moaA}$ 22094752209690 mopII $35529553554439 \mathrm{mpl}$ 11945341196264 nadE $35641443565196 \mathrm{nrdB}$ 29947862995391 orn 36303483632054 paaZ 11231321124643 pepA 23122212313243 pheS 23097012312133 pheT 34154553416225 phnK 35862383587455 pilC 27079662708541 ppiA 15900231590916 pstA 16498621653932 purL 34320383433120 queA 31487013149837 ribB $29540132955020 \mathrm{rluC}$ $29343652935768 \mathrm{rnc}$ $37951813796158 \mathrm{rpoA}$ 23266132327317 scoA $23259702326611 \mathrm{scoB}$ 960680961636 scrK 14425041443778 serA 318749321172 silP 906374907540 sucC 907685908566 sucD 38285443829734 tuf $725701 \quad 727278$ ubiB 36447873645524 ung 597179600046 uvrA 29240912926328 uvrC 32071163208354 wbiA 32033533205077 wbiD 33578263359841 wcbA 33537013354849 wcbD 33427433343783 wcbL 33294773337117 wcbR 32097503210583 wzm $32629523265315 \times \mathrm{xdhB}$ 10189101020292 xseA

\begin{tabular}{|c|c|c|c|}
\hline 0.0491502 & 1 & 1161410 & 1161517 \\
\hline 0.0741427 & 1 & 419469 & 419549 \\
\hline 0.0193424 & 1 & 3016488 & 3016508 \\
\hline 0.13547 & 1 & 768350 & 768536 \\
\hline 0.227357 & 1 & 2959466 & 2959813 \\
\hline 1 & 1 & 2209475 & 2209690 \\
\hline 0.0606469 & 1 & 3553483 & 3553573 \\
\hline 0.104046 & 1 & 1194836 & 1195016 \\
\hline 0.0551331 & 1 & 3564267 & 3564325 \\
\hline 0.0396694 & 1 & 2995281 & 2995305 \\
\hline 0.140094 & 1 & 3631634 & 3631873 \\
\hline 0.169424 & 1 & 1124159 & 1124415 \\
\hline 0.092955 & 1 & 2312840 & 2312935 \\
\hline 0.0226151 & 1 & 2311675 & 2311730 \\
\hline 0.0363636 & 1 & 3416069 & 3416097 \\
\hline 0.10189 & 1 & 3586480 & 3587455 \\
\hline 0.488696 & 1 & 2707966 & 2708247 \\
\hline 0.335946 & 1 & 1590529 & 1590829 \\
\hline 0.0218673 & 1 & 1651298 & 1651387 \\
\hline 0.252311 & 1 & 3432248 & 3432521 \\
\hline 0.142606 & 1 & 3149217 & 3149379 \\
\hline 0.204568 & 1 & 2954752 & 2954958 \\
\hline 0.451889 & 1 & 2934673 & 2935620 \\
\hline 0.230297 & 1 & 3795193 & 3795418 \\
\hline 0.0170455 & 1 & 2326613 & 2326625 \\
\hline 0.304212 & 1 & 2326416 & 2326611 \\
\hline 0.145397 & 1 & 960680 & 960819 \\
\hline 0.022763 & 1 & 1442962 & 1442991 \\
\hline 0.0239373 & 1 & 320926 & 320984 \\
\hline 0.138937 & 1 & 907162 & 907324 \\
\hline 0.170261 & 1 & 908101 & 908251 \\
\hline 0.576471 & 1 & 3829224 & 3829578 \\
\hline 0.0412175 & 1 & 726217 & 726282 \\
\hline 0.0393487 & 1 & 3644996 & 3645025 \\
\hline 0.0272061 & 1 & 598748 & 598826 \\
\hline 0.0102816 & 1 & 2925285 & 2925308 \\
\hline 0.0961228 & 1 & 3207988 & 3208107 \\
\hline 0.0203016 & 1 & 3204166 & 3204201 \\
\hline 0.0173697 & 1 & 3359601 & 3359636 \\
\hline 0.101045 & 1 & 3353755 & 3353871 \\
\hline 0.414423 & 1 & 3342913 & 3343344 \\
\hline 0.0454188 & 1 & 3334070 & 3335025 \\
\hline 0.128451 & 1 & 3210004 & 3210111 \\
\hline 0.00888701 & 1 & 3263766 & 3263787 \\
\hline 0.0296671 & 1 & 1020146 & 1020187 \\
\hline
\end{tabular}


Table S6 List of genes belonging to chromosome II with a mean_depth > 1 


\begin{tabular}{|c|c|c|c|c|c|c|c|}
\hline $\mathrm{chr}$ & start & locus_tag & strand & gene_coverage 1 & mean_depth & eak_start & peak_end \\
\hline gi_52211453_emb_BX571966 & 2736052 & 2737575 BPSS2025 & - & 0.0144452 & 20006.9 & 2737553 & 2737575 \\
\hline gi_52211453_emb_BX571966 & 2433214 & 2434440 BPSS1774 & - & 0.33279 & 6295.87 & 2434032 & 2434440 \\
\hline gi_52211453_emb_BX571966 & 499290 & 499841 BPSS0357 & + & 1 & 6146.87 & 499290 & 499841 \\
\hline gi_52211453_emb_BX571966 & 2176601 & 2177170 BPSS1601 & . & 0.367311 & 1890.96 & 2176733 & 2177170 \\
\hline gi $52211453 \mathrm{emb}$ BX571966 & 2660333 & 2661316 BPSS1970 & + & 0.905392 & 1066.3 & 2660362 & 2661316 \\
\hline gi_52211453_emb_BX571966 & 2177487 & 2178593 pilT & + & 0.669078 & 529.072 & 2177487 & 2178227 \\
\hline gi_52211453_emb_BX571966 & 2859411 & 2860655 BPSS2111 & + & 0.391479 & 308.532 & 2859411 & 2859898 \\
\hline gi_52211453_emb_BX571966 & 1322012 & 1323271 BPSS1002 & - & 0.252581 & 205.305 & 1322953 & 1323271 \\
\hline gi_52211453_e & 1585839 & $1587188 \mathrm{BP}$ & - & 009 & 197.157 & 1586902 & 1587188 \\
\hline gi_52211453_emb_BX571966 & 2731210 & 2732691 BPSS2021 & - & 464 & 133.672 & 2732286 & 2732691 \\
\hline gi_52211453_emb_BX571966 & 72252 & 72812 BPSS0064 & - & 0.0642857 & 122.944 & 72252 & 72288 \\
\hline gi_52211453_emb_BX571966 & 578412 & 579629 BPSS0418 & + & 0.133936 & 99.2331 & 578817 & 578980 \\
\hline gi_52211453_emb_BX571966 & 2732831 & 2733736 BPSS2022 & + & 0.267403 & 92.0579 & 2732831 & 2733073 \\
\hline gi_52211453_emb_BX571966 & 1293562 & 1295175 BPSS0976 & + & 0.201488 & 79.2492 & 1294850 & 1295175 \\
\hline gi_52211453_emb_BX571966 & 782752 & 783867 braC & + & 722 & 76.6667 & 782752 & 782905 \\
\hline gi_52211453_emb_BX571966 & 902357 & 904294 BPSS0666 & - & 109 & 67.7989 & 904115 & 904294 \\
\hline gi_52211453_emb_BX571966 & 2524957 & 2526144 BPSS1860 & 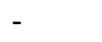 & 0.379949 & 61.0377 & 2525693 & 2526144 \\
\hline gi_52211453_emb_BX571966 & 1948688 & 1956592 BPSS1434 & + & 0.04542 & 54.5989 & 1948688 & 1949047 \\
\hline gi_52211453_e & 1295220 & 1296 & + & 717 & 50.5625 & 1295220 & 1295252 \\
\hline gi_52211453_e & 1003007 & $9 \mathrm{BPS}$ & - & 36 & 45.4246 & 1003109 & 1003865 \\
\hline gi_52211453_emb_BX571966 & 904497 & 905030 BPSS0667 & + & 223 & 43.9495 & 904497 & 904794 \\
\hline gi 52211453 emb BX571966 & 3143858 & 3146491 BPSS2330 & + & 0.0569692 & 43.4067 & 3145175 & 3145441 \\
\hline gi_52211453_c & 2795170 & 279 & + & 53 & 3376 & 170 & 404 \\
\hline gi_52211453_e & 1829021 & 1829 & - & 26 & 31.9158 & 1829021 & 1829306 \\
\hline gi_52211453_emb_BX571966 & 537046 & 537543 BPSS0391A & - & 219 & 30.4158 & 537442 & 537543 \\
\hline gi_52211453_emb_BX571966 & 2853944 & 2854462 BPSS2107 & - & 0.0810811 & 28 & 2854420 & 2854462 \\
\hline gi_52211453_emb_BX571966 & 547717 & $548649 \mathrm{BPS}$ & + & 476 & 27.2794 & 547717 & 548354 \\
\hline gi_52211453_e & 2855102 & 2855 & + & 696 & 26.7907 & 2855102 & 2855231 \\
\hline gi_52211453_emb_BX571966 & 2822906 & 2824966 BPS & - & 835 & 26.7862 & 2824475 & 2824966 \\
\hline gi_52211453_emb_BX571966 & 1556400 & 15572 & + & 142 & 26.5129 & 1556478 & 1557020 \\
\hline gi_52211453_emb_BX571966 & 947399 & 948070 BPSS0705 & + & 53 & 24.7512 & 947399 & 947600 \\
\hline gi_52211453_e & 546190 & 547 & + & 91 & 22.8577 & 547293 & 547560 \\
\hline gi_52211453_e & 2808477 & 2809 & - & 99 & 21.1588 & 2808913 & 2809190 \\
\hline gi_52211453_e & 3166744 & 6PSS2 & + & 65 & 20.2937 & 3166744 & 3166996 \\
\hline gi_52211453_en & 2793880 & 35 ans $A$ & - & 806 & 20.0104 & 2794164 & 2794935 \\
\hline gi_5221145 & 143 & 143 & - & & 577 & 147 & 14 \\
\hline gi_52211453_e & 836572 & $\mathrm{BPs}$ & - & 39 & 19.5794 & 836815 & 837426 \\
\hline gi_52211453_e & 496976 & $6 \mathrm{uvrB}$ & + & & 313 & 498449 & 499066 \\
\hline gi 52211453 emb BX571966 & 51665 & 5 chrA & - & 261 & 18.4711 & 52302 & 52855 \\
\hline gi_52211453_e & 1811570 & 1812 & - & & 17.6667 & 1811995 & 1812529 \\
\hline gi_52211453_ & 788473 & BPs & + & 44 & 16.0165 & 788473 & 788776 \\
\hline gi_52211453_emb_BX571966 & 54765 & 3 BPSS0055 & + & 555 & 15.9558 & 54765 & 55150 \\
\hline gi_52211453_emb_BX571966 & 2066773 & 56 BPSS1513 & + & 471 & 15.198 & 2066965 & 2067066 \\
\hline gi_52211453_emb_BX5 & 186668 & $3 \mathrm{BPs}$ & - & 174 & 15.0798 & 187108 & 189283 \\
\hline gi_52211453_e & 878644 & 878 & + & 1 & 145 & 878644 & 878913 \\
\hline gi_52211453_emb_BX571966 & 894966 & BPSS0659 & + & 23 & 14.3158 & 894966 & 895118 \\
\hline gi_52211453_emb_BX571966 & 2067071 & 2067703 folE & + & 0.639241 & 14.099 & 2067071 & 2067475 \\
\hline gi_5221145 & 1967536 & 1968 & - & 41 & 14.0099 & 1968465 & 1968667 \\
\hline gi_52211 & 1323383 & 1323 & - & 19 & 764 & 383 & 1323595 \\
\hline gi_52211453_emb_BX571966 & 1113438 & 5 BPSS0827 & + & 834 & 951 & 1113438 & 1114073 \\
\hline gi_52211453_emb_BX571966 & 1828597 & 1829031 BPSS1338 & - & 0.490783 & 12.8967 & 1828818 & 1829031 \\
\hline gi 5221145 & 2359545 & 2360 & - & 0.335 & 12.791 & 2359545 & 2359813 \\
\hline gi_52211453_emb_BX571966 & 946182 & $0 \mathrm{BPS}$ & - & 82721 & 12.7871 & 946636 & 947270 \\
\hline gi_52211453_emb_BX571966 & 2358929 & 2359345 BPSS1720 & - & 0.115385 & 12 & 2359297 & 2359345 \\
\hline gi_52211453_emb_BX571966 & 2262606 & 2263775 BPSS1646 & - & 0.420017 & 11.6619 & 2263224 & 2263715 \\
\hline gi_5221145 & 1645592 & 1647145 BPSS1218 & + & 0.457824 & 11.2729 & 1645810 & 1646521 \\
\hline gi_52211453_emb_BX571966 & 1960277 & 9 BPSS1439 & + & 0.07 & 11.0124 & 1960277 & 1960822 \\
\hline gi_52211453_emb_BX571966 & 2306033 & 2307187 BPSS1679 & + & 0.577123 & 10.8258 & 2306033 & 2307187 \\
\hline gi_52211453_emb_BX571966 & 2714171 & 2715988 glmS3 & - & 0.207485 & 10.4164 & 2714780 & 2715940 \\
\hline gi_52211453_emb_BX571966 & 110182 & 110727 BPSS0091 & + & 0.383486 & 10.4115 & 110182 & 110391 \\
\hline gi_5221145 & & $199391 \mathrm{BP}$ & + & 0.229545 & 9.62376 & 198951 & 199052 \\
\hline gi_52211453_emb_BX571966 & 522475 & 522720 BPSS0381 & + & 0.8 & 9.30612 & 522475 & 52267 \\
\hline
\end{tabular}


gi_52211453_emb_BX571966 gi_52211453_emb_BX571966 gi 52211453 emb BX571966 gi_52211453_emb_BX571966 gi 52211453 emb BX571966 gi 52211453 emb BX571966 gi_52211453_emb_BX571966 gi 52211453 emb BX571966 gi_52211453_emb_BX571966 gi_52211453_emb_BX571966 gi 52211453 emb BX571966 gi_52211453_emb_BX571966 gi_52211453_emb_BX571966 gi 52211453 emb_BX571966 gi_52211453_emb_BX571966 gi_52211453_emb_BX571966 gi 52211453 emb BX571966 gi_52211453_emb_BX571966 gi_52211453_emb_BX571966 gi_52211453_emb_BX571966 gi_52211453_emb_BX571966 gi_52211453_emb_BX571966 gi_52211453_emb_BX571966 gi_52211453_emb_BX571966 gi_52211453_emb_BX571966 gi_52211453_emb_BX571966 gi_52211453_emb_BX571966 gi_52211453_emb_BX571966 gi_52211453_emb_BX571966 gi_52211453_emb_BX571966 gi_52211453_emb_BX571966 gi_52211453_emb_BX571966 gi_52211453_emb_BX571966 gi_52211453_emb_BX571966 gi_52211453_emb_BX571966 gi_52211453_emb_BX571966 gi_52211453_emb_BX571966 gi_52211453_emb_BX571966 gi_52211453 emb_BX571966 gi_52211453_emb_BX571966 gi_52211453_emb_BX571966 gi 52211453 emb BX571966 gi_52211453_emb_BX571966 gi_52211453_emb_BX571966 gi 52211453 emb_BX571966 gi_52211453_emb_BX571966 gi_52211453_emb_BX571966 gi 52211453 emb BX571966 gi_52211453_emb_BX571966 gi_52211453_emb_BX571966 gi_52211453_emb_BX571966 gi_52211453_emb_BX571966 gi_52211453_emb_BX571966 gi_52211453_emb_BX571966 gi_52211453_emb_BX571966 gi_52211453_emb_BX571966 gi_52211453_emb_BX571966 gi_52211453_emb_BX571966 gi_52211453_emb_BX571966 gi_52211453_emb_BX571966 gi 52211453 emb_BX571966 gi_52211453_emb_BX571966 gi_52211453_emb_BX571966
888951890615 BPSS0655 28203422821583 BPSS2084 $1200501 \quad 1202816$ BPSS0908 14763801478893 BPSS1100 17585571759897 BPSS1287 26651242667058 BPSS1973 28216192822863 BPSS2085 $304350 \quad 305177$ BPSS0225 $88287 \quad 88592$ BPSS0077A + $532668 \quad 533243$ tnpR 495489496688 tyrB $603915 \quad 605813$ BPSS0441 12744241276016 BPSS0964 953131954333 BPSS0708 25124212514517 BPSS1850 19235071924622 BPSS1411

$91706 \quad 93946$ BPSS0079 $131327 \quad 135061$ BPSS0106 493316494287 BPSS0353 21805942181328 BPSS1604 $105290 \quad 107227$ BPSS0088 11725471173671 BPSS0879 24624312463285 BPSS1803 121159122508 BPSS0101 26874912689113 BPSS1987 $616361 \quad 617449$ BPSS0451 27029202705784 BPSS1998 28405422842050 BPSS2099 30180813020396 BPSS2246 28875712889091 BPSS2132 27983782799457 BPSS2064 151956152618 BPSS0119 649327651861 BPSS0479 99533101128 BPSS0086 18912151892744 BPSS1384 772931773881 BPSS0566 12701051273500 BPSS0962 31611203163402 BPSS2345 16633001663770 BPSS1231 395439396971 BPSS0294 12835731284991 BPSS0970 16441001644699 BPSS1217 10166471016949 BPSS0756 14997851500882 BPSS1118 29277552928762 BPSS2165 24180162418900 ispA 27707662780077 BPSS2053 $159632 \quad 160324$ BPSS0124 157968158624 BPSS0123 $892577 \quad 894607$ BPSS0658 769544771247 BPSS0564 901100901996 BPSS0665 909175910872 BPSS0671

$377013 \quad 378707$ BPSS0282

$639178 \quad 640512$ BPSS0469 $358089358871 \operatorname{argT}$

24216422422517 BPSS1767 $890647 \quad 891024$ BPSS0656 21735222174820 pilO $974797 \quad 975591$ BPSS0725 17952441796458 estA 23278162328310 BPSS1693 570903573890 BPSS0415
$+$

$-$

$+$

$+$$$
+
$$$$
+
$$$$
+
$$

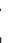$$
+
$$$$
-
$$$$
+
$$$$
-
$$

\begin{tabular}{|c|c|c|c|}
\hline 0.347957 & 344 & 888951 & 615 \\
\hline 0.134569 & 9.19162 & 2821416 & 2821583 \\
\hline 0.183585 & 9.05412 & 1200501 & 1200926 \\
\hline 0.181058 & 8.95165 & 1477157 & 1478743 \\
\hline 0.149254 & 8.345 & 1758557 & 1759168 \\
\hline 0.317477 & 8.20521 & 2665351 & 2666259 \\
\hline 0.406752 & 8.17194 & 2821619 & 2822125 \\
\hline 0.0749698 & 8 & 304350 & 304412 \\
\hline 0.318033 & 7.84536 & 88287 & 88384 \\
\hline 0.196522 & 7.69027 & 532668 & 532781 \\
\hline 0.168474 & 7.58416 & 495489 & 495691 \\
\hline 0.238145 & 7.54867 & 604525 & 604977 \\
\hline 0.384422 & 7.4085 & 1275404 & 1276016 \\
\hline 0.235441 & 7.39929 & 953131 & 953414 \\
\hline 0.263359 & 7.24094 & 2513965 & 2514517 \\
\hline 0.10852 & 7.2314 & 1924473 & 1924594 \\
\hline 0.212054 & 7.20421 & 92293 & 93946 \\
\hline 0.106588 & 7.07286 & 132027 & 132425 \\
\hline 0.0278064 & 7 & 494260 & 494287 \\
\hline 0.0408719 & 7 & 2180594 & 2180624 \\
\hline 0.284977 & 6.80616 & 105290 & 106389 \\
\hline 0.36 & 6.54126 & 1172547 & 1172959 \\
\hline 0.571429 & 6.44672 & 2462431 & 2462919 \\
\hline 0.444033 & 6.31553 & 121522 & 122121 \\
\hline 0.0357583 & 6.25862 & 2688919 & 2688977 \\
\hline 0.67 & $6.1 \mathrm{C}$ & 616 & 617449 \\
\hline 0.241271 & 6.17366 & 2704395 & 2705086 \\
\hline 0.192971 & 6.04124 & 2840990 & 2841281 \\
\hline 0.138229 & 5.8875 & 3019870 & 3020190 \\
\hline 0.4625 & 5.77667 & 2887571 & 2888952 \\
\hline 0.0639481 & 5.4 & 2799377 & 2799446 \\
\hline 0.60574 & 5.40648 & 151971 & 152372 \\
\hline 0.0745856 & 5.39683 & 649578 & 649767 \\
\hline 0.139185 & 5.39189 & 99619 & 99841 \\
\hline 0.17 & 5.3 & 1891897 & 1892708 \\
\hline 0.603158 & 5.35253 & 772931 & 773504 \\
\hline 0.492489 & 5.32656 & 1270171 & 1273196 \\
\hline 0.0714286 & 5.20245 & 3161938 & 3163342 \\
\hline 0.75 & 5.08 & 1663417 & 1663770 \\
\hline 0.0254569 & 5 & 395439 & 395478 \\
\hline 0.0874471 & 5 & 1284867 & 1284991 \\
\hline 0.320534 & 5 & 1644384 & 1644576 \\
\hline 0.31 & 4.88542 & 101 & 1016949 \\
\hline 0.289881 & 4.8239 & 1499785 & 1500263 \\
\hline 0.355511 & 4.78771 & 2928219 & 2928577 \\
\hline 0.466063 & 4.67961 & 2418362 & 2418774 \\
\hline 0.218022 & 4.63202 & 2772310 & 2778739 \\
\hline 0.426301 & 4.60678 & 160014 & 160309 \\
\hline 0.652439 & 4.58411 & 157968 & 158624 \\
\hline 0.253202 & 4.33852 & 892577 & 894607 \\
\hline 0.116853 & 4.28141 & 769615 & 769814 \\
\hline 0.524554 & 4.23191 & 901257 & 901786 \\
\hline 0.127873 & 4.21659 & 909704 & 909921 \\
\hline 0.0171192 & 4 & 377719 & 377748 \\
\hline 0.0262369 & 4 & 639994 & 640029 \\
\hline 0.122762 & 4 & 358430 & 358526 \\
\hline 0.328 & 3.95122 & 2422230 & 2422517 \\
\hline 0.734748 & 3.9278 & 890647 & 890924 \\
\hline 0.251156 & 3.85276 & 2174410 & 2174736 \\
\hline 0.15869 & 3.71429 & 975240 & 975366 \\
\hline 0.185338 & 3.71111 & 1795840 & 1796065 \\
\hline 0.398785 & 3.65482 & 2327816 & 2328013 \\
\hline 0.258788 & 708 & 1010 & 571783 \\
\hline
\end{tabular}


gi_52211453_emb_BX571966 gi_52211453_emb_BX571966 gi 52211453 emb BX571966 gi_52211453_emb_BX571966 gi 52211453 emb_BX571966 gi 52211453 emb BX571966 gi_52211453_emb_BX571966 gi 52211453 emb BX571966 gi 52211453 emb BX571966 gi_52211453_emb_BX571966 gi 52211453 emb BX571966 gi 52211453 emb BX571966 gi_52211453_emb_BX571966 gi 52211453 emb_BX571966 gi_52211453_emb_BX571966 gi_52211453_emb_BX571966 gi 52211453 emb BX571966 gi_52211453_emb_BX571966 gi_52211453_emb_BX571966 gi 52211453 emb_BX571966 gi_52211453_emb_BX571966 gi_52211453_emb_BX571966 gi_52211453_emb_BX571966 gi_52211453_emb_BX571966 gi_52211453_emb_BX571966 gi_52211453_emb_BX571966 gi_52211453_emb_BX571966 gi_52211453_emb_BX571966 gi_52211453_emb_BX571966 gi_52211453_emb_BX571966 gi_52211453_emb_BX571966 gi_52211453_emb_BX571966 gi_52211453_emb_BX571966 gi_52211453_emb_BX571966 gi_52211453_emb_BX571966 gi_52211453 emb_BX571966 gi_52211453_emb_BX571966 gi_52211453_emb_BX571966 gi 52211453 emb BX571966 gi_52211453_emb_BX571966 gi_52211453_emb_BX571966 gi 52211453 emb BX571966 gi_52211453_emb_BX571966 gi_52211453_emb_BX571966 gi 52211453 emb_BX571966 gi_52211453_emb_BX571966 gi_52211453_emb_BX571966 gi 52211453 emb BX571966 gi_52211453_emb_BX571966 gi_52211453_emb_BX571966 gi_52211453_emb_BX571966 gi_52211453_emb_BX571966 gi_52211453_emb_BX571966 gi_52211453_emb_BX571966 gi_52211453_emb_BX571966 gi_52211453_emb_BX571966 gi_52211453_emb_BX571966 gi_52211453_emb_BX571966 gi_52211453_emb_BX571966 gi_52211453_emb_BX571966 gi 52211453 emb_BX571966 gi_52211453_emb_BX571966 gi_52211453_emb_BX571966
14989631499634 BPSS1117 12125271213438 BPSS0919 10064751006846 BPSS0748 13262511327483 BPSS1005 401900403765 BPSS0299 18127701813786 BPSS1324 16263891628635 BPSS1204 13419491359456 BPSS1007 21787922180597 BPSS1603 16337101635902 BPSS1212 30920213094897 BPSS2299 $174740 \quad 175159$ BPSS0135 17553231757467 BPSS1285 $16499141650915 \mathrm{araF}$

26451872646128 BPSS1957 19264311927135 BPSS1414 23901902391284 lipA1

17580151758188 BPSS1286a 13043821305131 BPSS0986 $247624 \quad 248283$ BPSS0184 266228268741 BPSS0200 522852525719 BPSS0382 22692502269741 BPSS1650 31347873141608 BPSS2328 $44232 \quad 45629 \mathrm{pcaB}$ $365585 \quad 366787 \mathrm{pcaF}$ 21033292103631 BPSS1549 24381112438509 BPSS1778 23323982333246 BPSS1698 13160501317054 BPSS0996 11921361193809 BPSS0902 $898946 \quad 899656$ BPSS0664 10953271101467 BPSS0817 23650862367803 acnA 21093792111076 BPSS1555 410865423266 BPSS0306 25097342510318 BPSS1847 $312200 \quad 313861$ BPSS0229 25632712564527 BPSS1888 319052320464 BPSS0235 17970521798512 BPSS1312 27998692801308 BPSS2065 $18743641875749 \mathrm{sdaB}$ 10760621076697 BPSS0803 25446202546674 BPSS1873 29593832960573 BPSS2192 23166942318160 BPSS1685 12971911298306 BPSS0979 23680502370776 BPSS1727 27967042797141 BPSS2062 $392243 \quad 395428$ BPSS0293 18326961833628 BPSS1342 341809342303 BPSS0254 $73093 \quad 75045$ BPSS0065 27859282787196 BPSS2055 29333492934509 BPSS2169 15009331503989 BPSS1119 28570372858941 BPSS2110 20010962002322 BPSS1467 22668332269253 BPSS1649 960706961941 BPSS0713 18954311899264 BPSS1386 23916082393857 BPSS1742

\begin{tabular}{|c|c|c|c|}
\hline 0.272727 & 3.45902 & 1499451 & 1499634 \\
\hline 0.360044 & 3.45732 & 1212527 & 1213032 \\
\hline 0.784367 & 3.44674 & 1006532 & 1006823 \\
\hline 0.519481 & 3.44531 & 1326475 & 1327115 \\
\hline 0.251475 & 3.4371 & 402775 & 403244 \\
\hline 0.0935039 & 3.36842 & 1812803 & 1812898 \\
\hline 0.243099 & 3.3315 & 1626389 & 1627258 \\
\hline 0.024733 & 3.32564 & 1349107 & 1349540 \\
\hline 0.0221607 & 3.325 & 2180557 & 2180597 \\
\hline 0.0310219 & 3.32353 & 1635294 & 1635362 \\
\hline 0.102225 & 3.29252 & 3093457 & 3093751 \\
\hline 0.355609 & 3.26846 & 174870 & 175019 \\
\hline 0.0475746 & 3.2451 & 1757314 & 1757416 \\
\hline 0.283716 & 3.23239 & 1649914 & 1650198 \\
\hline 0.241233 & 3.22026 & 2645901 & 2646128 \\
\hline 0.747159 & 3.12167 & 1926527 & 1927053 \\
\hline 0.235832 & 3.1124 & 2391026 & 2391284 \\
\hline 0.462428 & 3.0875 & 1758108 & 1758188 \\
\hline 0.353805 & 3.0717 & 1304382 & 1304664 \\
\hline 0.517451 & 3 & 247656 & 247997 \\
\hline 0.0939117 & 3 & 268336 & 268572 \\
\hline 0.0153471 & 3 & 523511 & 523555 \\
\hline 0.0570265 & 3 & 2269250 & 2269278 \\
\hline 0.009676 & 3 & 3136748 & 3136814 \\
\hline 0.0171797 & 3 & 45121 & 45145 \\
\hline 0.120632 & 3 & 366025 & 366170 \\
\hline 1 & 2.9702 & 2103329 & 2103631 \\
\hline 0.947236 & 2.96552 & 2438111 & 2438497 \\
\hline 0.0860849 & 2.9589 & 2332486 & 2332559 \\
\hline 0.584661 & 2.82794 & 1316288 & 1316875 \\
\hline 0.272564 & 2.80921 & 1193176 & 1193750 \\
\hline 0.688732 & 2.80573 & 899167 & 899656 \\
\hline 0.0488599 & 2.76667 & 1096140 & 1096440 \\
\hline 0.380493 & 2.72948 & 2367369 & 2367503 \\
\hline 0.130819 & 2.72523 & 2109557 & 2109779 \\
\hline 0.025885 & 2.71651 & 411020 & 418659 \\
\hline 0.125 & 2.71233 & 2509740 & 2509813 \\
\hline 0.131848 & 2.70776 & 312316 & 312535 \\
\hline 0.0835987 & 2.69524 & 2563360 & 2563465 \\
\hline 0.43272 & 2.69394 & 319276 & 319887 \\
\hline 0.030137 & 2.68182 & 1797469 & 1797513 \\
\hline 0.0250174 & 2.66667 & 2799946 & 2799982 \\
\hline 0.101805 & 2.65248 & 1875608 & 1875749 \\
\hline 0.480315 & 2.65246 & 1076296 & 1076601 \\
\hline 0.0608569 & 2.624 & 2545476 & 2545601 \\
\hline 0.25042 & 2.60067 & 2959498 & 2959796 \\
\hline 0.130286 & 2.59686 & 2317149 & 2317340 \\
\hline 0.423318 & 2.57203 & 1297834 & 1298306 \\
\hline 0.12069 & 2.53799 & 2368884 & 2369213 \\
\hline 0.386728 & 2.52663 & 2796915 & 2797084 \\
\hline 0.264678 & 2.49822 & 392331 & 395428 \\
\hline 0.418455 & 2.47949 & 1832768 & 1833158 \\
\hline 0.182186 & 2.46667 & 342132 & 342222 \\
\hline 0.248975 & 2.43827 & 74559 & 75045 \\
\hline 0.0725552 & 2.42391 & 2785976 & 2786068 \\
\hline 0.262069 & 2.38816 & 2934205 & 2934509 \\
\hline 0.128272 & 2.38776 & 1501097 & 1503261 \\
\hline 0.0609244 & 2.37931 & 2858401 & 2858517 \\
\hline 0.323002 & 2.36616 & 2001926 & 2002322 \\
\hline 0.213636 & 2.34816 & 2267916 & 2269253 \\
\hline 0.208097 & 2.33463 & 960910 & 961167 \\
\hline 0.178711 & 2.32847 & 1897192 & 1899085 \\
\hline 0.108493 & 2.31557 & 2393218 & 2393462 \\
\hline
\end{tabular}


gi_52211453_emb_BX571966 gi_52211453_emb_BX571966 gi 52211453 emb BX571966 gi_52211453_emb_BX571966 gi 52211453 emb_BX571966 gi 52211453 emb BX571966 gi_52211453_emb_BX571966 gi 52211453 emb BX571966 gi 52211453 emb BX571966 gi_52211453_emb_BX571966 gi 52211453 emb BX571966 gi 52211453 emb BX571966 gi_52211453_emb_BX571966 gi 52211453 emb_BX571966 gi_52211453_emb_BX571966 gi_52211453_emb_BX571966 gi 52211453 emb BX571966 gi_52211453_emb_BX571966 gi_52211453_emb_BX571966 gi 52211453 emb_BX571966 gi_52211453_emb_BX571966 gi_52211453_emb_BX571966 gi_52211453_emb_BX571966 gi_52211453_emb_BX571966 gi_52211453_emb_BX571966 gi_52211453_emb_BX571966 gi_52211453_emb_BX571966 gi_52211453_emb_BX571966 gi_52211453_emb_BX571966 gi_52211453_emb_BX571966 gi_52211453_emb_BX571966 gi_52211453_emb_BX571966 gi_52211453_emb_BX571966 gi_52211453_emb_BX571966 gi_52211453_emb_BX571966 gi_52211453 emb_BX571966 gi_52211453_emb_BX571966 gi_52211453_emb_BX571966 gi_52211453 emb_BX571966 gi_52211453_emb_BX571966 gi_52211453_emb_BX571966 gi 52211453 emb BX571966 gi_52211453_emb_BX571966 gi_52211453_emb_BX571966 gi 52211453 emb_BX571966 gi_52211453_emb_BX571966 gi_52211453_emb_BX571966 gi 52211453_emb_BX571966 gi_52211453_emb_BX571966 gi_52211453_emb_BX571966 gi_52211453_emb_BX571966 gi_52211453_emb_BX571966 gi_52211453_emb_BX571966 gi 52211453 emb BX571966 gi_52211453_emb_BX571966 gi_52211453_emb_BX571966 gi_52211453_emb_BX571966 gi_52211453_emb_BX571966 gi_52211453_emb_BX571966 gi_52211453_emb_BX571966 gi 52211453 emb_BX571966 gi_52211453_emb_BX571966 gi_52211453_emb_BX571966
11983901199796 BPSS0907 10738601074297 BPSS0801 $1800598 \quad 1803501$ iles 25472242548900 BPSS1875 980533982194 BPSS0731 11040951106299 BPSS0820 885526886344 BPSS0652A + 10633331068294 BPSS0796 22254942238816 BPSS1633 $542523 \quad 543698$ BPSS0402 26932482695182 BPSS1992 18359681838085 BPSS1346 23182092320389 BPSS1686 29545062955888 BPSS2188 29642442965221 BPSS2197 17514861752982 BPSS1282 $85817 \quad 86671$ BPSS0076 134954136312 BPSS0108 479646480593 BPSS0346 $681027 \quad 683675$ BPSS0500 854697855791 BPSS0626 923681924037 BPSS0683 929989930711 BPSS0688 10123281012648 BPSS0753 10126731014904 BPSS0754 10208171022049 BPSS0760 10731311073745 BPSS0800 11404201141766 BPSS0851 14327041432970 BPSS1053 14970781498832 BPSS1116 15361821536955 BPSS1148 16103991619863 BPSS1197 20337842035334 BPSS1492 20360342037980 BPSS1493 23097452310872 BPSS1681 25218092523041 BPSS1857 25770692578016 BPSS1900 26418772643280 BPSS1955 26891102690126 BPSS1988 29037232905414 BPSS2145 31533663154073 BPSS2338 20937552095062 bsaS $14171051420338 \mathrm{czcA}$ $22876792288593 \mathrm{fdoH}$ $22870562287682 \mathrm{fdoI}$ 18683941868855 folB $936566 \quad 937414 \mathrm{hpcB}$ 11432691144201 BPSS0853 10767551077132 BPSS0804 115675117351 BPSS0096 10777781079844 BPSS0805 $550540 \quad 562935$ BPSS0409 28052392806207 BPSS2070 15935441594350 BPSS1187 756804758072 BPSS0554 786264787037 livG 22552432256574 cat 2 10047581005855 BPSS0747 27681492769918 BPSS2052 27438822745498 BPSS2031 $847418 \quad 848212$ BPSS0621 16914271693619 BPSS1249 19437611944660 BPSS1429
$+$

$+$

$-$
$+$$$
+
$$

$+$

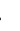

$+$

$-$

$+$

$-$

$-$
0.197724

0.354691

0.0120565

0.0369928

0.0716436

0.225953

0.183374

0.0290264

0.0827203

0.32766

0.0594623

0.31129

0.0811927

0.237337

0.16479

0.11631

0.748244

0.0154639

0.0908131

0.0162387

0.351005

0.120787

0.0512465

0.1125

0.0233079

0.101461

0.166124

0.106241

0.221805

0.13797

0.0336352

0.00750211

0.0593548

0.0400822

0.210293

0.137987

0.291447

0.0791162

0.0748031

0.148433

0.0367751

0.141546

0.00556758

0.0185996

0.255591

0.281996

0.123821

0.196352

0.413793

0.153938

0.0561471

0.0761597

0.357438

0.168734

0.318612

0.080207

0.114951

0.0865998

0.308649

0.112624

0.175063

0.0364964

0.057842

2.22662

2.2

2.2

2.19355

2.19328

2.17269

2.16667

2.16667

2.15608

2.10649

2.10435

2.10167

2.09605

2.09146

2.0559

2.03448

2.01252

\section{2}

2
2

2

\section{2}

2

2

2

2

2

\section{2}


gi_52211453_emb_BX571966 gi_52211453_emb_BX571966 gi_52211453_emb_BX571966 gi_52211453_emb_BX571966 gi 52211453 emb_BX571966 gi_52211453_emb_BX571966 gi_52211453_emb_BX571966 gi 52211453 emb BX571966 gi_52211453_emb_BX571966 gi_52211453_emb_BX571966 gi 52211453 emb BX571966 gi_52211453_emb_BX571966 gi_52211453_emb_BX571966 gi_52211453_emb_BX571966 gi_52211453_emb_BX571966 gi_52211453_emb_BX571966 gi_52211453_emb_BX571966 gi_52211453_emb_BX571966 gi_52211453_emb_BX571966 gi_52211453_emb_BX571966 gi_52211453_emb_BX571966 gi_52211453_emb_BX571966 gi_52211453_emb_BX571966 gi_52211453_emb_BX571966 gi_52211453_emb_BX571966 gi_52211453_emb_BX571966 gi_52211453_emb_BX571966 gi_52211453_emb_BX571966 gi_52211453_emb_BX571966 gi_52211453_emb_BX571966 gi_52211453_emb_BX571966 gi_52211453_emb_BX571966 gi_52211453_emb_BX571966 gi_52211453_emb_BX571966 gi_52211453_emb_BX571966 gi_52211453_emb_BX571966 gi_52211453_emb_BX571966 gi_52211453_emb_BX571966 gi_52211453 emb_BX571966 gi_52211453_emb_BX571966 gi_52211453_emb_BX571966 gi_52211453_emb_BX571966 gi_52211453_emb_BX571966 gi_52211453_emb_BX571966 gi_52211453_emb_BX571966 gi_52211453_emb_BX571966 gi_52211453_emb_BX571966 gi_52211453_emb_BX571966 gi_52211453_emb_BX571966 gi_52211453_emb_BX571966 gi_52211453_emb_BX571966 gi_52211453_emb_BX571966 gi_52211453_emb_BX571966 gi_52211453_emb_BX571966 gi_52211453_emb_BX571966 gi_52211453_emb_BX571966 gi_52211453_emb_BX571966 gi_52211453_emb_BX571966 gi_52211453_emb_BX571966 gi_52211453_emb_BX571966 gi 52211453 emb_BX571966 gi_52211453_emb_BX571966 gi_52211453_emb_BX571966
25207362521812 BPSS1856 29222102923289 BPSS2160 16320331633571 BPSS1211 12507561251091 BPSS0949 17857061786626 BPSS1305 $34692 \quad 35642$ BPSS0035 29934732994231 BPSS2219 542377542526 BPSS0401 217017217988 BPSS0164 14257801426265 BPSS1046 11683991169010 BPSS0875 975934977442 BPSS0726 17906411791687 BPSS1308 607554608903 BPSS0443 741664743229 BPSS0543 208096210993 BPSS0159 $580405 \quad 581478 \mathrm{rfbG}$

27927312793330 BPSS2058 10220491023305 codA

28702092871330 BPSS212 1 $667638 \quad 669245 \mathrm{ahpF}$

28312272833431 BPSS2093

$1743703 \quad 1744773 \mathrm{cnrT}$

428856437384 BPSS0311

29134352914022 BPSS2153

$86668 \quad 87699$ BPSS0077

9997771001294 BPSS0743

23708772372568 BPSS1728

$834728 \quad 836518$ BPSS0612

22775262279721 bglB

$157098 \quad 157745$ BPSS0122

26243602625484 BPSS1939

23978722398204 BPSS1748

$13107671312284 \mathrm{katB}$

$177878 \quad 178165$ BPSS0137

10389841039424 BPSS0778

$31521673152919 \mathrm{glnH}$

23220382323081 gca

485360486852 BPSSr01

529868532291 BPSS0385

22388132248688 BPSS1634

26578332658741 BPSS1968

15447241548527 narG

$658624 \quad 662499$ BPSS0486

15133241514727 BPSS1128

19197311920690 BPSS1406

820930821088 BPSS0599

998211999566 BPSS0742

20440522045794 BPSS1500

29084782909989 BPSS2149

16668951667707 BPSS1234

13052491305593 BPSS0987

29652762966235 BPSS2198

170998171984 BPSS0131

932953933390 BPSS0691

487486490367 BPSSr02

14482901449165 BPSS1077

$56852 \quad 62767$ uvrA

22886042291675 fdoG

22072132225497 BPSS1632

12438851245039 BPSS0943

944313946082 BPSS0703 21339082134765 tauC

\begin{tabular}{|c|c|c|c|}
\hline 0.202602 & 1.95872 & 2521339 & 2521812 \\
\hline 0.109361 & 1.95763 & 2922210 & 2922328 \\
\hline 0.0149545 & 1.95652 & 1632357 & 1632380 \\
\hline 0.241791 & 1.92593 & 1251010 & 1251091 \\
\hline 0.356522 & 1.92073 & 1786145 & 1786473 \\
\hline 0.0484211 & 1.91304 & 34765 & 34811 \\
\hline 0.0751979 & 1.91228 & 2994174 & 2994231 \\
\hline 0.758389 & 1.90265 & 542413 & 542526 \\
\hline 0.0267765 & 1.88462 & 217590 & 217616 \\
\hline 0.725773 & 1.875 & 1425780 & 1426198 \\
\hline 0.535188 & 1.86239 & 1168540 & 1168925 \\
\hline 0.0855438 & 1.85271 & 976061 & 976190 \\
\hline 0.250478 & 1.85115 & 1791325 & 1791587 \\
\hline 0.209044 & 1.84397 & 607873 & 608809 \\
\hline 0.149521 & 1.83761 & 742995 & 743229 \\
\hline 0.0800828 & 1.8319 & 210258 & 210964 \\
\hline 0.44548 & 1.83054 & 580778 & 581364 \\
\hline 0.223706 & 1.82836 & 2793193 & 2793327 \\
\hline 0.334395 & 1.81667 & 1022049 & 1023226 \\
\hline 0.131133 & 1.80272 & 2870235 & 2870382 \\
\hline 0.288114 & 1.77754 & 667799 & 668262 \\
\hline 0.1951 & 1.77209 & 2831796 & 2832226 \\
\hline 0.0607477 & 1.75385 & 1744533 & 1744598 \\
\hline 0.0259146 & 1.74661 & 428986 & 429831 \\
\hline 0.115843 & 1.73529 & 2913879 & 2913947 \\
\hline 0.29001 & 1.72575 & 86668 & 87063 \\
\hline 0.320369 & 1.72016 & 1000494 & 1000980 \\
\hline 0.077469 & 1.71756 & 2371836 & 2371967 \\
\hline 0.127374 & 1.71491 & 835472 & 835700 \\
\hline 0.1959 & 1.71163 & 2277526 & 2279575 \\
\hline 0.347759 & 1.71111 & 157189 & 157745 \\
\hline 0.022242 & 1.68 & 2625459 & 2625484 \\
\hline 0.225904 & 1.66667 & 2398037 & 2398112 \\
\hline 0.255109 & 1.65375 & 1310857 & 1311374 \\
\hline 0.463415 & 1.64662 & 177878 & 178011 \\
\hline 0.443182 & 1.6359 & 1039172 & 1039367 \\
\hline 0.5625 & 1.59574 & 3152496 & 3152919 \\
\hline 0.511026 & 1.59099 & 2322186 & 2322916 \\
\hline 0.671582 & 1.59082 & 485543 & 486852 \\
\hline 0.143211 & 1.57637 & 530437 & 532291 \\
\hline 0.051443 & 1.56299 & 2240664 & 2242711 \\
\hline 0.269824 & 1.5551 & 2658121 & 2658366 \\
\hline 0.171444 & 1.55215 & 1545210 & 1547694 \\
\hline 0.0856774 & 1.54819 & 660577 & 661692 \\
\hline 0.0798289 & 1.54464 & 1513546 & 1513658 \\
\hline 0.308655 & 1.54054 & 1920267 & 1920563 \\
\hline 0.582278 & 1.51087 & 820930 & 821022 \\
\hline 0.139483 & 1.50265 & 998885 & 999074 \\
\hline 0.316877 & 1.47826 & 2044899 & 2045451 \\
\hline 0.25546 & 1.4715 & 2908720 & 2909106 \\
\hline 0.402709 & 1.46177 & 1667143 & 1667470 \\
\hline 0.25 & 1.44186 & 1305507 & 1305593 \\
\hline 0.260688 & 1.436 & 2965276 & 2966213 \\
\hline 0.0567951 & 1.41071 & 171022 & 171755 \\
\hline 0.356979 & 1.35897 & 933234 & 933390 \\
\hline 0.459216 & 1.35752 & 488097 & 490201 \\
\hline 0.198857 & 1.35632 & 1448991 & 1449165 \\
\hline 0.0101437 & 1.35 & 58040 & 58737 \\
\hline 0.27255 & 1.34528 & 2290484 & 2291321 \\
\hline 0.04627 & 1.33806 & 2213047 & 2217623 \\
\hline 0.354419 & 1.33252 & 1243916 & 1244325 \\
\hline 0.107971 & 1.32461 & 945646 & 945837 \\
\hline 0.276546 & 30802 & 21343 & 213456 \\
\hline
\end{tabular}


gi_52211453_emb_BX571966 gi_52211453_emb_BX571966 gi 52211453 emb BX571966 gi_52211453_emb_BX571966 gi 52211453 emb BX571966 gi 52211453 emb BX571966 gi_52211453_emb_BX571966 gi 52211453 emb BX571966 gi 52211453 emb_BX571966 gi_52211453_emb_BX571966 gi 52211453 emb BX571966 gi_52211453_emb_BX571966 gi_52211453_emb_BX571966 gi 52211453 emb_BX571966 gi_52211453_emb_BX571966 gi_52211453_emb_BX571966 gi 52211453 emb BX571966 gi_52211453_emb_BX571966 gi_52211453_emb_BX571966 gi_52211453_emb_BX571966 gi_52211453_emb_BX571966 gi_52211453_emb_BX571966 gi_52211453_emb_BX571966 gi_52211453_emb_BX571966 gi_52211453_emb_BX571966 gi_52211453_emb_BX571966 gi_52211453_emb_BX571966 gi_52211453_emb_BX571966 gi_52211453_emb_BX571966 gi_52211453_emb_BX571966 gi_52211453_emb_BX571966 gi_52211453_emb_BX571966 gi_52211453_emb_BX571966 gi_52211453_emb_BX571966 gi_52211453_emb_BX571966 gi_52211453_emb_BX571966 gi_52211453_emb_BX571966 gi_52211453_emb_BX571966 gi_52211453_emb_BX571966 gi_52211453_emb_BX571966 gi_52211453_emb_BX571966 gi 52211453 emb BX571966 gi_52211453_emb_BX571966 gi_52211453_emb_BX571966 gi 52211453 emb_BX571966 gi_52211453_emb_BX571966 gi_52211453_emb_BX571966 gi 52211453 emb BX571966 gi_52211453_emb_BX571966 gi_52211453_emb_BX571966 gi_52211453_emb_BX571966 gi_52211453_emb_BX571966 gi_52211453_emb_BX571966 gi_52211453_emb_BX571966 gi_52211453_emb_BX571966 gi_52211453_emb_BX571966 gi_52211453_emb_BX571966 gi_52211453_emb_BX571966 gi_52211453_emb_BX571966 gi_52211453_emb_BX571966 gi 52211453 emb_BX571966 gi_52211453_emb_BX571966 gi_52211453_emb_BX571966
19765031978822 BPSS1450 + $3722 \quad 4921 \mathrm{kbl}+$

213569215074 BPSS0162 +

19382341939232 BPSS1424

24232372425030 BPSS1768

476085477194 BPSS0343

14308951432688 BPSS1052

110792111529 BPSS0092

27014642702273 oxa

27101292710617 BPSS2003

19994172000928 BPSS1466

587459588715 BPSS0425

14873681488162 BPSS1109

29559342956779 BPSS2189

23635872365038 prpD

20842592085518 bipC

10079351009344 BPSS0750

28928812894323 BPSS2136

26518692653143 BPSS1963

17213871734097 BPSS1269

562919566497 BPSS0410

$37431 \quad 38354$ BPSS0038

$55704 \quad 56372$ BPSS0057

$84913 \quad 85833$ BPSS0075

9425995614 BPSS0080

109043109597 BPSS0090

$111753 \quad 114386$ BPSS0093

127919128488 BPSS0104

165876167096 BPSS0129

$168157 \quad 170964$ BPSS0130

203913204674 BPSS0156

$221397 \quad 225473$ BPSS0167

241527243854 BPSS0181

$248330 \quad 248704$ BPSS0185

249406251322 BPSS0186

$256752 \quad 257597$ BPSS0192

330905333184 BPSS0244

$354335 \quad 355450$ BPSS0267

400571401470 BPSS0298

408326409807 BPSS0304

423325424737 BPSS0307

439636440475 BPSS0313

440493441887 BPSS0314

454103454540 BPSS0325

$456588 \quad 457880$ BPSS0327

474336475682 BPSS0342

477432478514 BPSS0344

$481481 \quad 482752$ BPSS0348

482758484203 BPSS0349

$505308 \quad 506279$ BPSS0365

$541537 \quad 542235$ BPSS0400

574629574985 BPSS0416A +

$575855 \quad 578407$ BPSS0417

593393594292 BPSS0431

596139597170 BPSS0434

628847630139 BPSS0461

$670272 \quad 671369$ BPSS0493

708199709188 BPSS0521

719014720087 BPSS0526

720090720806 BPSS0527

739867741531 BPSS0542

743418744434 BPSS0544

777815778123 BPSS0571

$\begin{array}{rr}0.114705 & 1.29699 \\ 0.206005 & 1.291 \\ 0.192691 & 1.2827 \\ 0.151303 & 1.2781 \\ 0.0663692 & 1.260 \\ 0.174031 & 1.25389 \\ 0.0440602 & 1.2405 \\ 0.213026 & 1.2356 \\ 0.343634 & 1.23022 \\ 0.270492 & 1.2272 \\ 0.0986102 & 1.2080 \\ 0.104299 & 1.2061 \\ 0.580605 & 1.19523 \\ 0.518343 & 1.15982 \\ 0.457615 & 1.15813 \\ 0.43606 & 1.12022 \\ 0.142654 & 1.1194 \\ 0.244105 & 1.11364 \\ 0.463108 & 1.11017 \\ 0.0403619 & 1.07407 \\ 0.148127 & 1.05094 \\ 0.0628386 & \\ 0.366766 & \end{array}$

.29699

1977866 3995

214538

1939081

$2423822 \quad 2423941$

$476915 \quad 477108$

$1432353 \quad 1432688$

$111098 \quad 111255$

$\begin{array}{ll}2701506 & 2701784\end{array}$

$2710129 \quad 2710515$

$2000778 \quad 2000927$

$588055 \quad 588186$

$1487445 \quad 1487906$

29559342956372

$2363701 \quad 2364365$

$2084334 \quad 2084883$

$1008417 \quad 1008618$

$2893971 \quad 2894323$

$2652425 \quad 2653015$

$1723080 \quad 1730490$

$563283 \quad 565261$

$37967 \quad 38025$

$55751 \quad 56372$

$85650 \quad 85700$

$95260 \quad 95289$

$109154 \quad 109333$

$114029 \quad 114101$

$\begin{array}{ll}128332 & 128488\end{array}$

$166837 \quad 167033$

$168766 \quad 168966$

$204024 \quad 204375$

$222468 \quad 224270$

$241636 \quad 241835$

$248662 \quad 248692$

$251190 \quad 251322$

$256870 \quad 256913$

$332159 \quad 332361$

$354930 \quad 355340$

$400621 \quad 400870$

$409487 \quad 409713$

$424196 \quad 424406$

$439720 \quad 440091$

$440663 \quad 440680$

$454103 \quad 454377$

$457193 \quad 457785$

$474611 \quad 474929$

$477542 \quad 477868$

$482694 \quad 482752$

$482758 \quad 482831$

$505910 \quad 506063$

$542058 \quad 542109$

$574899 \quad 574985$

$576912 \quad 577122$

$593739 \quad 593791$

$596310 \quad 596343$

$628847 \quad 628892$

$671073 \quad 671333$

$708696 \quad 708724$

$\begin{array}{ll}719598 & 719784\end{array}$

$720138 \quad 720382$

$741199 \quad 741531$

$743731 \quad 744035$

$777943 \quad 778077$ 
gi_52211453_emb_BX571966 gi_52211453_emb_BX571966 gi 52211453 emb BX571966 gi_52211453_emb_BX571966 gi 52211453 emb_BX571966 gi 52211453 emb BX571966 gi_52211453_emb_BX571966 gi 52211453 emb BX571966 gi 52211453 emb BX571966 gi_52211453_emb_BX571966 gi 52211453 emb BX571966 gi 52211453 emb BX571966 gi_52211453_emb_BX571966 gi 52211453 emb_BX571966 gi_52211453_emb_BX571966 gi_52211453_emb_BX571966 gi 52211453 emb BX571966 gi_52211453_emb_BX571966 gi_52211453_emb_BX571966 gi_52211453_emb_BX571966 gi_52211453_emb_BX571966 gi_52211453_emb_BX571966 gi_52211453_emb_BX571966 gi_52211453_emb_BX571966 gi_52211453_emb_BX571966 gi_52211453_emb_BX571966 gi 52211453_emb_BX571966 gi_52211453_emb_BX571966 gi_52211453_emb_BX571966 gi_52211453_emb_BX571966 gi_52211453_emb_BX571966 gi_52211453_emb_BX571966 gi_52211453_emb_BX571966 gi_52211453_emb_BX571966 gi_52211453_emb_BX571966 gi_52211453_emb_BX571966 gi_52211453_emb_BX571966 gi_52211453_emb_BX571966 gi 52211453 emb BX571966 gi_52211453_emb_BX571966 gi_52211453_emb_BX571966 gi_52211453_emb_BX571966 gi_52211453_emb_BX571966 gi_52211453_emb_BX571966 gi 52211453 emb_BX571966 gi_52211453_emb_BX571966 gi_52211453_emb_BX571966 gi 52211453 emb BX571966 gi_52211453_emb_BX571966 gi_52211453_emb_BX571966 gi_52211453_emb_BX571966 gi_52211453_emb_BX571966 gi_52211453_emb_BX571966 gi 52211453 emb BX571966 gi_52211453_emb_BX571966 gi_52211453_emb_BX571966 gi_52211453_emb_BX571966 gi_52211453_emb_BX571966 gi_52211453_emb_BX571966 gi_52211453_emb_BX571966 gi 52211453 emb_BX571966 gi_52211453_emb_BX571966 gi_52211453_emb_BX571966
819701819895 BPSS0597+ 828849830321 BPSS0607 831174832406 BPSS0609 832403833314 BPSS0610 $856170 \quad 856892$ BPSS0627 925685926899 BPSS0685 972923973993 BPSS0723 10507071051531 BPSS0785 10517201052733 BPSS0786 10710341072254 BPSS0799 11036281104098 BPSS0819 11072191108121 BPSS0822 11360371137353 BPSS0847 11665071167709 BPSS0873 12071531208454 BPSS0913 12556611257262 BPSS0954 12639511268576 BPSS0960 12810701283394 BPSS0969 13075411308083 BPSS0989 13280911341815 BPSS1006 13993611400365 BPSS1028 14285121428727 BPSS1048c 14475941448124 BPSS1076 14502761451181 BPSS1080 14511741451728 BPSS1081 14517391454102 BPSS1082 14548461456018 BPSS1084 14882291489377 BPSS1110 15041241505662 BPSS1120 15090341510017 BPSS1124 15621221564590 BPSS1170 16005841605287 BPSS1193 16093121610373 BPSS1196 16517821653533 BPSS1222 17054921706649 BPSS1259 17165731719788 BPSS1266 17475741749181 BPSS1279 17499351750861 BPSS1281 17543041755326 BPSS1284 17726101773047 BPSS1294 17749021775570 BPSS1296 18585361860014 BPSS1358 18864751888661 BPSS1381 18886581889674 BPSS1382 18895571890045 BPSS1383 18998461901654 BPSS1388 19393551940257 BPSS1425 19809621984240 BPSS1453 19842271985591 BPSS1454 19890471990465 BPSS1458 19958531998192 BPSS1464 20387112040555 BPSS1495 20457822046804 BPSS1501 20467912049829 BPSS 1502 20498562052879 BPSS1503 20529052055547 BPSS1504 20651852066609 BPSS 1512 20800972082739 BPSS1528 21030002103269 BPSS1548 21036362104583 BPSS1550 21045802105167 BPSS1551 21455602150062 BPSS1580 21865732188018 BPSS1610
0.546392

0.0326087

0.0681818

0.169045

0.390582

0.053542

0.317757

0.059466

0.0394867

0.291803

0.0531915

0.0254989

0.0547112

0.0515807

0.0245965

0.0468457

0.0862703

0.230207

0.337638

0.0121685

0.0458167

0.181395

0.609434

0.241989

0.106498

0.0592467

0.0460751

0.103659

0.020156

0.207528

0.0628039

0.011482

0.213949

0.0148487

0.0613656

0.0650078

0.196017

0.277538

0.0225049

0.235698

0.296407

0.196888

0.0704483

0.253937

0.102459

0.241704

0.322616

0.161074

0.0307918

0.0282087

0.0175289

0.200651

0.0596869

0.10632

0.0605359

0.016654

0.0224719

0.0363361

0.130112

0.0770855

0.0749574

0.0424256

0.141869

819789

829504

832062

833021

856175

926076

973017

1051316

1051736

1071405

1103965

1107339

1136522

1166507

1207716

1256127

1265614

1281115

1307900

1338475

1399699

1428590

1447675

1450408

1451191

1453424

1455447

1488970

1504613

1509375

1563660

1603242

1610048

1652816

1706271

1719537

1748335

1750083

1754431

1772679

1775295

1858902

1887187

1889349

1889557

1900151

1939606

1983284

1985495

1989221

1997485

2039442

2046743

2046791

2052615

2054205

2065543

2081222

12103234

819895

829552

832146

833175

856457

926141

973357

1051365

1051776

1072108

1103990

1107362

1136594

1166569

1207748

1256202

1266013

1282166

1308083

1341021

1399745

1428629

1447998

1450627

1451250

1453564

1455501

1489089

1504644

1509579

1563815

1603296

1610275

1652842

1706342

1719746

1748864

1750340

1754454

1772782

1775493

1859193

1887341

1889607 
gi_52211453_emb_BX571966 gi_52211453_emb_BX571966 gi 52211453 emb BX571966 gi_52211453_emb_BX571966 gi 52211453 emb_BX571966 gi 52211453 emb_BX571966 gi_52211453_emb_BX571966 gi 52211453 emb BX571966 gi 52211453 emb BX571966 gi_52211453_emb_BX571966 gi 52211453 emb BX571966 gi 52211453 emb BX571966 gi_52211453_emb_BX571966 gi 52211453 emb_BX571966 gi_52211453_emb_BX571966 gi_52211453_emb_BX571966 gi 52211453 emb BX571966 gi_52211453_emb_BX571966 gi_52211453_emb_BX571966 gi_52211453_emb_BX571966 gi_52211453_emb_BX571966 gi_52211453_emb_BX571966 gi_52211453_emb_BX571966 gi_52211453_emb_BX571966 gi_52211453_emb_BX571966 gi_52211453_emb_BX571966 gi_52211453_emb_BX571966 gi_52211453_emb_BX571966 gi_52211453_emb_BX571966 gi_52211453_emb_BX571966 gi_52211453_emb_BX571966 gi_52211453_emb_BX571966 gi_52211453_emb_BX571966 gi_52211453_emb_BX571966 gi_52211453_emb_BX571966 gi_52211453 emb_BX571966 gi_52211453_emb_BX571966 gi_52211453_emb_BX571966 gi_52211453 emb_BX571966 gi_52211453_emb_BX571966 gi_52211453_emb_BX571966 gi 52211453 emb BX571966 gi_52211453_emb_BX571966 gi_52211453_emb_BX571966 gi 52211453 emb_BX571966 gi_52211453_emb_BX571966 gi_52211453_emb_BX571966 gi 52211453 emb BX571966 gi_52211453_emb_BX571966 gi_52211453_emb_BX571966 gi 52211453 emb BX571966 gi_52211453_emb_BX571966 gi_52211453_emb_BX571966 gi_52211453_emb_BX571966 gi_52211453_emb_BX571966 gi_52211453_emb_BX571966 gi_52211453_emb_BX571966 gi_52211453_emb_BX571966 gi_52211453_emb_BX571966 gi_52211453_emb_BX571966 gi 52211453 emb_BX571966 gi_52211453_emb_BX571966 gi_52211453_emb_BX571966
22022632202790 BPSS1628 22489892251772 BPSS1635 23009322301483 BPSS1675 23373842338700 BPSS1703 23528892353278 BPSS1713 24009132401851 BPSS1751 24485632450620 BPSS1790 24681552468997 BPSS1809 24839802485164 BPSS1826 25035072504775 BPSS1841 25402792541307 BPSS1870 25413142543908 BPSS1871 25792912580205 BPSS1902 25865402587592 BPSS1910 26173802618246 BPSS1935 26361552636856 BPSS1949 26559612656866 BPSS1966 26587382660336 BPSS1969 26678592669577 BPSS1974 27217512722743 BPSS2014 27475082749520 BPSS2034 27578412759694 BPSS2042 27670102768149 BPSS2051 27812832785914 BPSS2054 28062282807226 BPSS2071 28140542816087 BPSS2080 28334282836094 BPSS2094 28375482839419 BPSS2096 28555582856994 BPSS2 109 28859622886252 BPSS2131 28867102887267 BPSS2131b 29015072903009 BPSS2143 29057202906661 BPSS2146 29199962920745 BPSS2158 29308112932001 BPSS2167 29417252942777 BPSS2175 29447852945741 BPSS2177 29509752952216 BPSS2184 29605972961139 BPSS2193 29762632977177 BPSS2207 29942902995033 BPSS2220 30426813044030 BPSS2265 30734343075266 BPSS2285 30844373085498 BPSS2292 30875333088552 BPSS2295 30957663096716 BPSS2301 31065263107698 BPSS2306 31284483129149 BPSS2325 31291493130636 BPSS2326 31306693134790 BPSS2327 10403821040466 BPSSt04 669449670012 ahpC $24364292437955 \mathrm{amn}$ $21500592151579 \mathrm{bcsZ}$ $1849107 \quad 1850576$ betB 21005652101206 bsaN $20986892100551 \mathrm{bsaO}$ 25679622568864 catA $52852 \quad 53847 \mathrm{chrB}$ 25702532572265 cyoB 31577463158651 estC $11600561161558 \mathrm{feaB}$ 23291622330475 folC
0.0664137

0.0459935

0.0598911

0.0744681

0.262211

0.385928

0.133204

0.174584

0.066723

0.160095

0.07393

0.0289129

0.16302

0.0912548

0.0334873

0.108417

0.0475138

0.0281602

0.0291036

0.153226

0.13171

0.0906638

0.196664

0.0611099

0.0440882

0.0905066

0.110653

0.00908605

0.0473538

0.175862

0.181329

0.121838

0.398512

0.148198

0.156303

0.0836502

0.222803

0.13618

0.107011

0.11488

0.0121131

0.266123

0.120087

0.0263902

0.247301

0.231579

0.056314

0.154066

0.0322798

0.0361563

0.124334

0.177588

0.109211

0.0707965

0.0390016

0.146617

0.189579

0.115578

0.128231

0.038674

0.231025

0.0441736 $\begin{array}{ll}2202620 & 2202655\end{array}$

$2249695 \quad 2249823$

$1 \quad 2301314 \quad 2301347$

$1 \quad 2338251 \quad 2338349$

$1 \quad 2352889 \quad 2352991$

$1 \quad 2400988 \quad 2401350$

$1 \quad 2449724 \quad 2449998$

$\begin{array}{lll}1 & 2468850 & 2468997\end{array}$

$1 \quad 2484712 \quad 2484791$

$12503623 \quad 2503826$

$1 \quad 2541013 \quad 2541089$

$1 \quad 2542927 \quad 2543002$

$\begin{array}{lll}1 & 2579479 & 2580017\end{array}$

$\begin{array}{lll}1 & 2586868 & 2586964\end{array}$

$1 \quad 2617380 \quad 2617409$

$12636626 \quad 2636702$

$12656662 \quad 2656705$

$12659943 \quad 2659988$

$12668062 \quad 2668112$

$12722006 \quad 2722158$

$\begin{array}{lll}1 & 2748348 & 2748613\end{array}$

$\begin{array}{lll}1 & 2759523 & 2759691\end{array}$

$1 \quad 2767372 \quad 2767596$

$1 \quad 2782063 \quad 2782346$

$12806521 \quad 2806565$

$12815336 \quad 2815520$

$\begin{array}{lll}1 & 2835196 & 2835491\end{array}$

$\begin{array}{lll}1 & 2838427 & 2838444\end{array}$

$1 \quad 2856089 \quad 2856157$

$1 \quad 2885962 \quad 2886013$

$1 \quad 2886962 \quad 2887063$

$1 \quad 2901719 \quad 2901902$

$1 \quad 2905937 \quad 2906312$

$1 \quad 29204312920542$

$1 \quad 2931104 \quad 2931290$

$1 \quad 2941868 \quad 2941956$

$1 \quad 2945162 \quad 2945526$

$1 \quad 2951598 \quad 2951767$

$\begin{array}{lll}1 & 2960614 & 2960997\end{array}$

$1 \quad 2977004 \quad 2977109$

$1 \quad 2994290 \quad 2994299$

$13042955 \quad 3043314$

$\begin{array}{lll}1 & 3073859 & 3074079\end{array}$

$13085145 \quad 3085173$

$13088248 \quad 3088500$

$13096415 \quad 3096635$

$13107632 \quad 3107698$

$1 \quad 3129041 \quad 3129149$

$1 \quad 3129149 \quad 3129197$

$13133630 \quad 3133779$

$1 \quad 1040382 \quad 1040466$

$\begin{array}{lll}1 & 669504 & 669574\end{array}$

$1 \quad 2437594 \quad 2437955$

$1 \quad 2150382 \quad 2150548$

$\begin{array}{lll}1 & 1849673 & 1849777\end{array}$

$12100643 \quad 2100668$

$1 \quad 2098769 \quad 2099042$

$1 \quad 2568036 \quad 2568207$

$1 \quad 52852 \quad 52967$

$\begin{array}{lrr}1 & 2570829 & 2571087\end{array}$

$1 \quad 3157959 \quad 3157994$

$1 \quad 1160964 \quad 1161454$

$\begin{array}{lll}1 & 2329710 & 2329768\end{array}$ 
gi_52211453_emb_BX571966 gi 52211453 emb BX571966 gi_52211453_emb_BX571966 gi_52211453_emb_BX571966 gi 52211453 emb BX571966 gi_52211453_emb_BX571966 gi_52211453_emb_BX571966 gi 52211453 emb BX571966 gi_52211453_emb_BX571966 gi_52211453_emb_BX571966 gi 52211453 emb BX571966 gi_52211453_emb_BX571966 gi_52211453_emb_BX571966 gi 52211453 emb BX571966 gi_52211453_emb_BX571966 gi_52211453_emb_BX571966 gi 52211453 emb BX571966 gi_52211453_emb_BX571966 gi_52211453_emb_BX571966 gi_52211453_emb_BX571966 gi_52211453_emb_BX571966 gi_52211453_emb_BX571966 gi_52211453_emb_BX571966 gi_52211453_emb_BX571966 gi_52211453_emb_BX571966 gi_52211453_emb_BX571966 gi_52211453_emb_BX571966 gi_52211453_emb_BX571966 gi_52211453_emb_BX571966 gi_52211453_emb_BX571966 gi_52211453_emb_BX571966 gi_52211453_emb_BX571966 gi_52211453_emb_BX571966 gi_52211453_emb_BX571966 gi_52211453_emb_BX571966 gi_52211453_emb_BX571966 gi_52211453_emb_BX571966
$880135 \quad 881091 \mathrm{ftr} A$ $14936931495693 \mathrm{ftsH}$ $375588 \quad 376871$ gabT 23984032399818 gnd 24689942469938 ilvE $23891522390186 \mathrm{lipB}$ 28755832876725 lldA

$844881 \quad 846410 \mathrm{mmsA}$ $26954822696984 \mathrm{mprA}$ 15431981544727 narH 28975342898472 oppB 28956292896618 oppD 28944992895632 oppF 985782987641 oprM $1781580 \quad 1782173 \mathrm{pcaG}$ $17808731781577 \mathrm{pcaH}$ $800407 \quad 806454 \mathrm{pchF}$ 21748322176601 pilN 21703352171411 pilR 24269712429742 polA $636535 \quad 637656$ potF $633288 \quad 634217$ potI $278428 \quad 279321$ prpB 23262282327763 purF $344068 \quad 345045 \mathrm{rbsC}$ $581486 \quad 582838 \mathrm{rfbH}$ $29913022992777 \mathrm{rpoN}$ $19037321905498 \mathrm{sctC}$ $19208331921882 \mathrm{sctD}$ $23567762358551 \mathrm{sdh} A$ 22844742285916 selA $22825522284477 \mathrm{selB}$ 18694801872488 soxA 21355652136575 tauA $23332562334449 \operatorname{trpB}$ 10823881083590 tyrB 1 24927042494125 udg2
0.117155

0.0165

0.183944

0.134276

0.127119

0.0686654

0.0402802

0.150425

0.177097

0.211249

0.0426439

0.372093

0.00617829

0.0188273

0.0860034

0.205966

0.00446502

0.0825325

0.0167286

0.0187658

0.197145

0.106566

0.0257559

0.120521

0.126919

0.309911

0.0454237

0.168743

0.187798

0.0490141

0.0208044

0.0763636

0.00631649

0.293069

0.152557

0.0574043

0.100633
$880870 \quad 880982$

$1494859 \quad 1494892$

$375588 \quad 375824$

$2398403 \quad 2399753$

$2468994 \quad 2469114$

$2389227 \quad 2389298$

$2875894 \quad 2875940$

$844896 \quad 845126$

$2696025 \quad 2696291$

$1543897 \quad 1544220$

$2898138 \quad 2898178$

$2895820 \quad 2896188$

28944992894506

$987451 \quad 987486$

$1781814 \quad 1781865$

$1781206 \quad 1781351$

$804920 \quad 804947$

$\begin{array}{ll}2175217 & 2175363\end{array}$

$2170335 \quad 2170353$

$2428215 \quad 2429529$

$\begin{array}{ll}637378 & 637599\end{array}$

$633697 \quad 633796$

$279203 \quad 279226$

$2327419 \quad 2327604$

$344788 \quad 344912$

$581776 \quad 582195$

$2992101 \quad 2992168$

$1905117 \quad 1905450$

$1921675 \quad 1921872$

$2357294 \quad 2357381$

$2285464 \quad 2285494$

$2283736 \quad 2283883$

$1872219 \quad 1872238$

$\begin{array}{ll}2136279 & 2136575\end{array}$

$\begin{array}{ll}2333366 & 2333548\end{array}$

$1083444 \quad 1083513$

$\begin{array}{lll}1 & 2492885 & 2493028\end{array}$ 
Table S7 List of genes belonging to the three functional categories that were significantly enriched (pvalue $<0.01$ and $p$-value $<0.05$ ) in the ORF-filtered library

\begin{tabular}{|c|c|c|c|c|c|c|}
\hline \multicolumn{7}{|c|}{ Functional category: Inorganic ion transport and metabolism } \\
\hline locus_ID & strand & gene_coverage & mean_depth & peak_start & peak_end & gene description \\
\hline BPSL0466 & + & 1 & 54,3301 & 503530 & 504348 & ABC-type metal ion transport system, periplasmic component/surface antigen \\
\hline BPSL0882 & - & 0,248963 & 25,6933 & 1023539 & 1023839 & Chromate transport protein $\mathrm{ChrA}$ \\
\hline BPSL2351 & + & 0,189685 & 16,2189 & 2843904 & 2844338 & Nitric oxide reductase large subunit \\
\hline BPSL0824 & - & 0,008 & 14 & 958876 & 958883 & ABC-type metal ion transport system, periplasmic component/surface adhesin \\
\hline BPSL2368 & + & 0,359532 & 13,9442 & 2863293 & 2863723 & Uncharacterized protein involved in response to NO \\
\hline BPSL3404 & - & 0,338836 & 12,7974 & 4042163 & 4042553 & $\mathrm{Na}+/ \mathrm{H}+$ antiporter $\mathrm{NhaD}$ and related arsenite permeases \\
\hline BPSL0825 & - & 0,575431 & 10,5993 & 959141 & 959408 & $\mathrm{Fe} 2+/ \mathrm{Zn} 2+$ uptake regulation proteins \\
\hline BPSL2726 & - & 0,450128 & 7,8267 & 3262216 & 3262568 & ABC-type molybdate transport system, periplasmic component \\
\hline BPSL1001 & + & 0,131725 & 6,77465 & 1164821 & 1164892 & $\mathrm{Cu} / \mathrm{Zn}$ superoxide dismutase \\
\hline BPSL1824 & - & 0,564054 & 6,74407 & 2171943 & 2172533 & ABC-type nitrate/sulfonate/bicarbonate transport systems, periplasmic components \\
\hline BPSL0309 & + & 0,130448 & 6,58711 & 327333 & 330545 & Putative silver efflux pump \\
\hline BPSL3329 & + & 0,379699 & 5,76485 & 3948556 & 3948960 & $\begin{array}{l}\text { Phenylpropionate dioxygenase and related ring-hydroxylating dioxygenases, large } \\
\text { terminal subunit }\end{array}$ \\
\hline BPSL3007 & + & 0,400772 & 5,13487 & 3584331 & 3584850 & Putative $\mathrm{Mg} 2+$ and $\mathrm{Co} 2+$ transporter $\mathrm{CorB}$ \\
\hline BPSL0284 & + & 0,717863 & 4,07674 & 297641 & 298084 & Chromate transport protein $\mathrm{ChrA}$ \\
\hline BPSL1770 & - & 0,206409 & 4 & 2089749 & 2089968 & High-affinity nickel permease \\
\hline BPSL1554 & - & 0,122418 & 3,8875 & 1803765 & 1804075 & $\mathrm{Mn} 2+$ and $\mathrm{Fe} 2+$ transporters of the NRAMP family \\
\hline BPSL2849 & - & 0,443216 & 3,64399 & 3411823 & 3412264 & ABC-type phosphate/phosphonate transport system, periplasmic component \\
\hline BPSL2307 & + & 0,138281 & 3,34463 & 2784060 & 2784237 & Nitrate/nitrite transporter \\
\hline BPSL1839 & - & 0,0335707 & 3 & 2193983 & 2194016 & ABC-type sulfate transport system, periplasmic component \\
\hline BPSS0357 & + & 1 & 6146,87 & 499290 & 499841 & Uncharacterized protein probably involved in high-affinity $\mathrm{Fe} 2+$ transport \\
\hline BPSS1178 & - & 0,212009 & 197,157 & 1586902 & 1587188 & Kef-type $\mathrm{K}+$ transport systems, membrane components \\
\hline BPSS0404 & + & 0,194891 & 22,8577 & 547293 & 547560 & Cytochrome c peroxidase \\
\hline BPSS0053 & - & 0,451261 & 18,4711 & 52302 & 52855 & Chromate transport protein ChrA \\
\hline BPSS0704 & - & 0,582721 & 12,7871 & 946636 & 947270 & $\mathrm{ABC}$-type $\mathrm{Fe} 3+$ transport system, periplasmic component \\
\hline BPSS1100 & - & 0,181058 & 8,95165 & 1477157 & 1478743 & Cation transport ATPase \\
\hline BPSS1005 & - & 0,519481 & 3,44531 & 1326475 & 1327115 & Kef-type K+ transport systems, membrane components \\
\hline BPSS1204 & + & 0,243099 & 3,3315 & 1626389 & 1627258 & Outer membrane receptor for monomeric catechols \\
\hline \multicolumn{7}{|c|}{ Functional category: Nucleotide transport and metabolism } \\
\hline locus_ID & strand & gene_coverage & mean_depth & peak_start & peak_end & gene description \\
\hline BPSL1866 & + & 0,213035 & 31,242 & 2219853 & 2220072 & Dihydroorotate dehydrogenase \\
\hline BPSL1681 & + & 0,32491 & 18,7022 & 1962097 & 1962547 & Dihydroorotase and related cyclic amidohydrolases \\
\hline BPSL2896 & + & 0,208946 & 6,01223 & 3465705 & 3466032 & $\begin{array}{l}\text { AICAR transformylase/IMP cyclohydrolase PurH (only IMP } \\
\text { cyclohydrolase domain in Aful) }\end{array}$ \\
\hline BPSL0801 & + & 0,496656 & 5,67003 & 929168 & 929843 & Phosphoribosylaminoimidazole carboxylase (NCAIR synthetase) \\
\hline BPSL2116 & - & 0,452122 & 5,36026 & 2542885 & 2543343 & Allantoicase \\
\hline BPSL2928 & + & 0,0303239 & 5,18182 & 3497572 & 3497616 & Adenylosuccinate lyase \\
\hline BPSL3422 & + & 0,0792453 & 4,85714 & 4065048 & 4065090 & Adenylate cyclase, class 2 (thermophilic) \\
\hline BPSL1136 & - & 0,0850144 & 4,32203 & 1321632 & 1322113 & Xanthine/uracil permeases \\
\hline BPSL3167 & - & 0,0592532 & 3 & 3771626 & 3771699 & dGTP triphosphohydrolase \\
\hline BPSS0479 & + & 0,0745856 & 5,39683 & 649578 & 649767 & Ribonucleotide reductase, alpha subunit \\
\hline BPSS0045 & + & 0,0171797 & 3 & 45121 & 45145 & Adenylosuccinate lyase \\
\hline
\end{tabular}




\begin{tabular}{|c|c|c|c|c|c|c|}
\hline \multicolumn{7}{|c|}{ Functional category: Cell envelope biogenesis, outer membrane } \\
\hline locus_ID & strand & gene_coverage & mean_depth & peak_start & peak_end & gene description \\
\hline BPSL2559 & + & 0,341528 & 63,9126 & 3087604 & 3088394 & Outer membrane protein (porin) \\
\hline BPSL2199 & - & 0,850214 & 48,4648 & 2640323 & 2640919 & Cell wall-associated hydrolases (invasion-associated proteins) \\
\hline BPSL3036 & + & 0,357759 & 18,012 & 3617871 & 3618286 & Outer membrane protein (porin) \\
\hline BPSL0338 & - & 0,348607 & 13,3252 & 360861 & 361599 & Phospholipase C \\
\hline BPSL1674 & - & 0,337273 & 10,0135 & 1953804 & 1954175 & Outer membrane protein (porin) \\
\hline BPSL1029 & - & 0,108182 & 9,31933 & 1199418 & 1199537 & Outer membrane protein (porin) \\
\hline BPSL3249 & + & 0,428916 & 9,2809 & 3861407 & 3861763 & Outer membrane protein $\mathrm{W}$ \\
\hline BPSL0408 & + & 0,223782 & 6,87823 & 443603 & 443874 & D-alanyl-D-alanine carboxypeptidase \\
\hline BPSL2152 & - & 0,179008 & 6,51807 & 2582595 & 2582844 & Predicted membrane-associated $\mathrm{Zn}$-dependent proteases 1 \\
\hline BPSL0307 & + & 0,269024 & 6,37429 & 324700 & 325050 & Outer membrane protein \\
\hline BPSL2297 & - & 0,134384 & 6 & 2765011 & 2765156 & D-alanyl-D-alanine carboxypeptidase \\
\hline BPSL3161 & + & 0,573187 & 5,09547 & 3765568 & 3765987 & Outer membrane protein $\mathrm{W}$ \\
\hline BPSL2151 & - & 0,389779 & 4,95667 & 2581630 & 2582530 & Outer membrane protein/protective antigen OMA87 \\
\hline BPSL1655 & + & 0,242397 & 4,24723 & 1917834 & 1918105 & Outer membrane protein (porin) \\
\hline BPSL0308 & + & 0,189052 & 4,14478 & 327039 & 327336 & Membrane-fusion protein \\
\hline BPSL0442 & - & 0,257942 & 4,0936 & 482402 & 483709 & Periplasmic protease \\
\hline BPSL2179 & - & 0,00186916 & 4 & 2617410 & 2617412 & Alanine racemase \\
\hline BPSL0183 & - & 0,0350163 & 3,81395 & 187471 & 187557 & Cell division protein FtsI/penicillin-binding protein 2 \\
\hline BPSL1345 & + & 0,130644 & 3,45161 & 1569365 & 1569582 & Soluble lytic murein transglycosylase and related regulatory proteins \\
\hline BPSL0725 & + & 0,0672727 & 3,13514 & 834804 & 834878 & $\begin{array}{l}\text { L-alanine-DL-glutamate epimerase and related enzymes of enolase } \\
\text { superfamily }\end{array}$ \\
\hline BPSL0590 & - & 0,0841674 & 3 & 656517 & 657922 & Rhs family protein \\
\hline BPSS0418 & + & 0,133936 & 99,2331 & 578817 & 578980 & Capsule polysaccharide export protein \\
\hline BPSS0580 & + & 0,245344 & 16,0165 & 788473 & 788776 & Membrane proteins related to metalloendopeptidases \\
\hline BPSS1679 & + & 0,577123 & 10,8258 & 2306033 & 2307187 & Outer membrane protein (porin) \\
\hline BPSS2009 & - & 0,207485 & 10,4164 & 2714780 & 2715940 & $\begin{array}{l}\text { Glucosamine 6-phosphate synthetase, contains amidotransferase and } \\
\text { phosphosugar isomerase domains }\end{array}$ \\
\hline BPSS1287 & + & 0,149254 & 8,345 & 1758557 & 1759168 & Outer membrane protein \\
\hline BPSS0708 & + & 0,235441 & 7,39929 & 953131 & 953414 & Outer membrane protein (porin) \\
\hline BPSS0879 & + & 0,366548 & 6,54126 & 1172547 & 1172959 & Outer membrane protein (porin) \\
\hline BPSS0451 & - & 0,671875 & 6,19836 & 616714 & 617449 & D-alanyl-D-alanine carboxypeptidase \\
\hline BPSS2064 & - & 0,0639481 & 5,49275 & 2799377 & 2799446 & Outer membrane protein (porin) \\
\hline BPSS0294 & + & 0,0254569 & 5 & 395439 & 395478 & Outer membrane protein \\
\hline BPSS1118 & + & 0,289881 & 4,8239 & 1499785 & 1500263 & Membrane-fusion protein \\
\hline BPSS2165 & + & 0,355511 & 4,78771 & 2928219 & 2928577 & Nucleoside-diphosphate-sugar epimerases \\
\hline
\end{tabular}



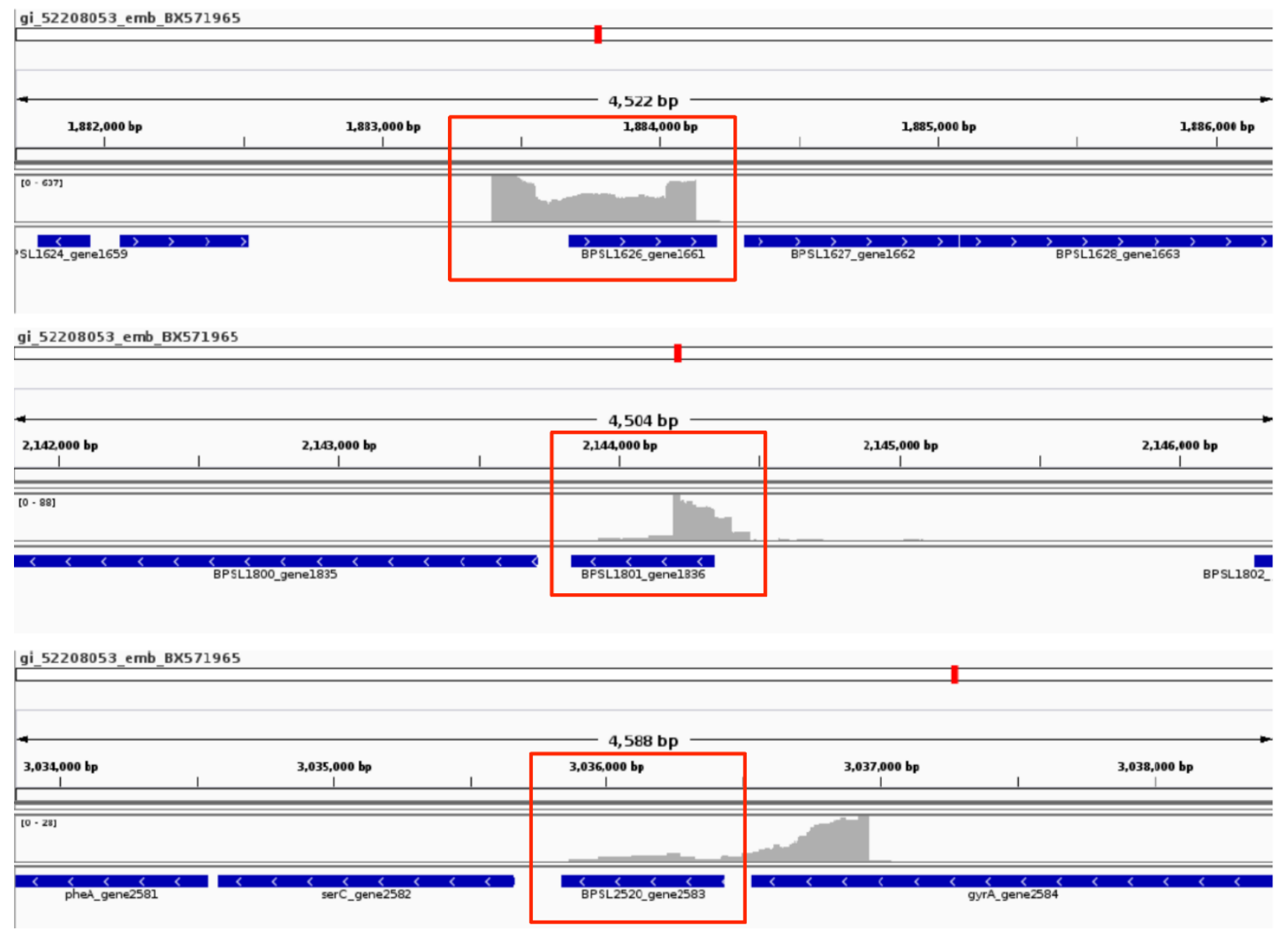

Figure S1 Integrated Genomics Viewer visualization of sequencing reads mapping on the genes of BPSL1626, BPSL1801 and BPSL252 